\title{
Swissness als Erfolgsfaktor - Einsatz des Country-of-Origin zur Stärkung von Marken-Konsumenten-Beziehungen und der Markenbindung
}
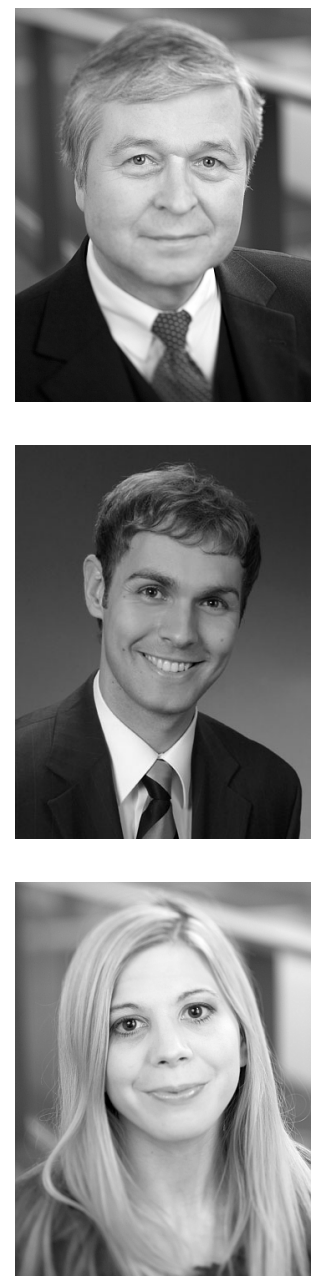
elements of brand consumer relationship - brand satisfaction, brand commitment, brand trust - and brand loyalty. The developed research model is tested with the brand Ricola.

\section{Manfred Brubn, Jürgen Schwarz und Verena Batt}

Country-of-Origin, Country-of-Origin-Effekt, Marken-Konsumenten-Beziehung, Markenzufriedenheit, Markencommitment, Markenvertrauen, Markenbindung.

Country-of-Origin, Country-of-Origin-Effect, Brand Consumer Relationship, Brand Satisfaction, Brand Commitment, Brand Trust, Brand Loyalty.

Im Rahmen der Forschung zum Country-of-Origin (COO) bleibt bislang die Frage unbeantwortet, welche Massnahmen von Unternehmen einzusetzen sind, damit die Herkunft einer Marke überhaupt von Konsumenten wahrgenommen wird und welche Wirkungen das $\mathrm{COO}$ auf psychologische und verhaltensbezogene Grössen hat. In der vorliegenden Untersuchung wird die Schweiz als COO herangezogen. Es wird ein Strukturgleichungsmodell entwickelt, das zum einen Rückschlüsse auf die Qualität der kommunikativen Übertragung des COO zulässt und zum anderen die Wirkung des $\mathrm{COO}$ auf die zentralen Grössen der Marken-Konsumenten-Beziehung - Markenzufriedenheit, Markencommitment und Markenvertrauen - analysiert. Die empirische Überprüfung des Strukturgleichungsmodells erfolgt am Beispiel der Marke Ricola.

Regarding the research about country-of-origin (COO) the question which measures marketing managers should implement in order to ensure that brand origin is perceived by consumers, still remain unanswered. Moreover, a large number of the studies remark on the lack of attention paid to psychological and behaviour-related effects. In this paper, Switzerland is chosen as COO. The present paper aims to develop a model with the capacity to draw conclusions about the determinants of the COO effect and, thus, also about its transmission as well as about the influence of the COO on central 


\section{Relevanz des Country-of-Origin und Stand der Forschung}

Die Bedeutung von starken Marken für den Unternehmenserfolg wurde in der Marketingwissenschaft u. a. über deren präferenzprägende Funktion für Konsumenten ausreichend nachgewiesen (u. a. Fournier 1998; Chaudhuri/Holbrook 2002; Bengtsson 2003; Madden et al. 2006). Im Rahmen der Markenpolitik befassen sich Forscher in den letzten Jahren vermehrt mit Marken-Konsumenten-Beziehungen (u. a. Fournier/Yao 1997; Fournier 1998; Iacobucci/Hibbard 1999; Bengtsson 2003; Veloutsou 2007). Diese Entwicklung basiert vorwiegend auf dem Wandel vom Transaktions- zum Beziehungsmarketing. Der Wandel zu einer beziehungsorientierten Markenpolitik wird durch die Erkenntnis begründet, dass erfolgswirksame Effekte, wie z. B. eine höhere Kundenloyalität oder ein positives Weiterempfehlungsverhalten, eine beziehungsorientierte Ausrichtung sämtlicher Marketingaktivitäten eines Unternehmens benötigen (u. a. Anderson/Weitz 1992; Garbarino/Johnson 1999; Dibb/Meadows 2004; Gounans 2005). Die Entwicklung von Marken-Konsumenten-Beziehungen erfordert dabei ein bestimmtes Mass an Aktivität einer Marke. Die einzelnen Marketingmassnahmen werden von Konsumenten als Handlungen einer Marke interpretiert und daraus Rückschlüsse auf die Markenpersönlichkeit gezogen (Fournier 1998, 348).

Neben markenbezogenen Marketingaktivitäten werden konsumentenseitige Markenassoziationen zusätzlich von unternehmensexternen Einflussfaktoren, wie z. B. dem Country-of-Origin (COO) bestimmt (u. a. Abmed et al. 2004; Michaelis et al. 2008; Lee/Lee 2009). Durch die Übertragung von Eigenschaften des COO auf eine Marke wird aus Konsumentensicht die Markenpersönlichkeit beeinflusst und detaillierter wahrgenommen. Das Herkunftsland dient beispielsweise im Rahmen des Kaufentscheidungsprozesses von Konsumenten als Qualitätshinweis und zur Risikoreduktion (Cordell 1992, 251). Konsumenten berücksichtigen das $\mathrm{COO}$ bei Kaufentscheidungen, wenn nicht genügend Informationen für eine individuelle Markenbewertung vorhanden sind oder die existierenden Angaben über Marken nur schwer zu vergleichen und einzuschätzen sind (Thorelli et al. 1989, 43). Vor allem bei der Gegenüberstellung internationaler Marken wird das COO als Persönlichkeitswert einer Marke wahrgenommen und als Ersatz für fehlendes Wissen über Qualität und Leistungseigenschaften genutzt (Parameswaran/Pisharodi 1994, 44). Es liegt daher nahe, dass in Zeiten der zunehmenden Internationalisierung von Unternehmensaktivitäten das Herkunftsland als ein wesentlicher Wettbewerbsvorteil anzusehen ist und einen positiven Einfluss auf die Markenwahrnehmung und dementsprechend auf Marken-Konsumenten-Beziehungen ausübt. Durch die Verknüpfung des COO mit positiven Markenassoziationen besteht für Unternehmen die Möglichkeit, ihre Marken von der Konkurrenz $\mathrm{zu}$ differenzieren und die Marken-Konsumenten-Beziehung zu verbessern (Balabanis/ Diamantopoulos 2011).

Die Untersuchung des aktuellen Forschungsstands zum COO offenbart die Existenz zahlreicher empirischer und konzeptioneller Arbeiten. Daher werden die Literatursynopse von Bilkey und Nes (1982) sowie die Meta-Analyse von Verlegh und Steenkamp (1999) als Ausgangspunkt herangezogen. Nach Bilkey und Nes (1982) bestätigen mehrere Studien den Einfluss des $\mathrm{COO}$ auf die konsumentenseitige Wahrnehmung von Produkten sowie deren Einstellung. Zudem überprüfen Studien den COO-Effekt in Abhängigkeit vom Entwicklungsstand eines Landes, den Einfluss des wahrgenommenen Kaufrisikos sowie demografischer und persönlicher Merkmale von Konsumenten und den Einfluss von COO bezogenen Marketingmassnahmen (Bilkey/Nes 1982). Die Meta-Analyse von Verlegh und 
Steenkamp (1999) fasst die Erkenntnisse von 41 empirischen Studien zusammen. Dabei werden der Einfluss des COO auf die wahrgenommene Produktqualität, die Abhängigkeit vom Entwicklungsstand eines Landes, der COO-Effekt bei mehrteiligen und einteiligen Produkten für Industrie- sowie Konsumgüter und der unterschiedliche Einfluss bei Multiattribut- und Einzelattribut-Modellen nachgewiesen. Letztlich weisen Studien einen signifikanten Unterschied zwischen Studenten- und Konsumentenbefragungen nach. Aufgrund der zahlreichen Beiträge zum COO werden in der nachfolgenden Betrachtung vorwiegend Artikel aus den vom Verband der Hochschullehrer für Betriebswirtschaft (VHB) gerankten A-, B- und C-Journals sowie die meist zitierten Studien über psychologische und verhaltensbezogene Wirkungsgrössen berücksichtigt.

Die Wirkung des COO auf die Produktevaluation untersuchen Hong und Wyer Jr. (1989), die insbesondere damit zusammenhängende kognitive Prozesse betrachten. Aus der Studie resultiert, dass das COO das Interesse von Konsumenten am Produkt anregt. In einer Folgestudie untersuchen Hong und Wyer Jr. (1990) den Einfluss der COO-Information, wenn diese zeitlich vor anderen Produktattributen bekannt ist oder zeitgleich vorgestellt wird. Die zeitlich vorgelagerte COO-Information beeinflusst die Produktevaluation stärker, da Konsumenten im Voraus ein Produktkonzept entwickeln, das dann durch weitere Produktattribute ergänzt wird. Liu und Johnson (2005) bestätigen ebenfalls bei zeitlich vorgelagerter Konfrontation mit der COO-Information einen positiven Effekt auf die Produktevaluation. Die Wirkung ist bei Impulskäufen grösser als bei langfristigen Kaufentscheidungen. Ebenso belegen Hastak und Hong (1991), unter Einbezug des Produktpreises, die positive Auswirkung des $\mathrm{COO}$ auf die konsumentenseitige Einschätzung der Produktqualität.

Elliott und Cameron (1994) vergleichen hingegen den Einfluss von Produktpreis und -qualität mit dem COO-Effekt und stellen fest, dass Konsumenten lokale Produkte bevorzugen, wenn keine Qualitäts- und Preisunterschiede bestehen. Darüber hinaus untersuchen Gürhan-Canli und Maheswaran (2000b) unter Berücksichtigung der Motivation für einen Produktkauf die Relevanz der COO-Information im Vergleich zu weiteren Produktattributen. Demnach ist die COO-Information von Bedeutung, wenn die Kaufmotivation gering ist und die Produktattribute unterschiedliche Produktteile betreffen. Eine weiterführende Studie von Maheswaran und Chen (2006) bestätigt den Einfluss der Gefühlslage von Konsumenten auf die Verwendung des COO zur Produktevaluation. Thakor und Lavack (2003) weisen den Einfluss der wahrgenommenen geografischen Markenherkunft auf die Bewertung funktionaler Nutzenkomponenten der Produktqualität nach. Ergänzend dazu bekräftigt die Studie von Batra et al. (2000) den Einfluss der wahrgenommenen Markenherkunft auf symbolische Nutzenkomponenten.

Aus der empirischen Studie von Erickson et al. (1984) geht hervor, dass COO-Informationen, trotz zusätzlich vorliegender Produktaspekte, einen signifikanten Einfluss auf die Einstellung und indirekt auf das Konsumentenverhalten ausüben. In einem weiterführenden Modell belegen Johansson et al. (1985) unter Einbezug demografischer Variablen und der Produktvertrautheit die Wirkung des COO auf die Bewertung einzelner Produktattribute. Den Einfluss der Produktvertrautheit auf den Zusammenhang zwischen COO und Produktevaluation untersuchen ebenso Heimbach et al. (1989). Dabei wird nachgewiesen, dass vorwiegend Produktvertraute auf das COO zur Produktbewertung zurückgreifen. Darauf aufbauend zeigt Han (1989), dass beim Erstkauf eines Produktes das COO aufgrund mangelnder Erfahrung über die Produktqualität herangezogen wird und für Pro- 
duktvertraute das COO hingegen als Gesamtbewertung des Produktes dient. Im Zusammenhang mit der Markenvertrautheit untersuchen Tse und Gorn (1993) das Zusammenspiel von COO und globalen Marken und stellen fest, dass sowohl die Herkunftsinformation als auch die Marke eine wichtige Rolle bei der Produktevaluation spielen. Mit zunehmender Interaktion zwischen Konsument und Marke weist das COO einen stärkeren Effekt auf als die Marke. Jiménez und San Martín (2010) belegen unter Berücksichtigung der psychologischen Variablen Ethnozentrismus und der Animosität der Konsumenten den COO-Effekt auf das Markenvertrauen.

Die Studie von Roth und Romeo (1992) analysieren den Fit zwischen Ländern und den dort hergestellten Produktkategorien. Die Autoren belegen den Einfluss des COO auf die Kaufabsicht, wenn ein Land in der Herstellung der Produkte als stark wahrgenommen wird. Li et al. (1994) identifizieren das COO, den Preis und die Marke als Treiber der Kaufabsicht, sofern diese vom Konsumenten positiv bewertet werden. Tabelle 1 gibt einen Überblick über sämtliche relevanten Studien.

Zusammenfassend ist zu konstatieren, dass der Schwerpunkt der vorgestellten Forschungsbeiträge auf dem Einfluss des $\mathrm{COO}$ auf die Produktevaluation und die Kaufabsicht gerichtet ist (u. a. Roth/Romeo 1992; Li et al. 1994; Abmed et al. 2004; Hong/Kang 2006). Dabei werden in einer Vielzahl von Studien unterschiedliche Einflussfaktoren und Moderatoren berücksichtigt, die Zielgrössen jedoch unverändert bei den zuvor genannten belassen.

Trotz zahlreicher Studien zum COO offenbaren sich zentrale Forschungsdefizite: Eine Vielzahl an Studien konstatiert die mangelnde Berücksichtigung von psychologischen und verhaltensbezogenen Wirkungsbeziehungen des COO-Effekts auf Markenebene (u. a. Abmed et al. 2004; Liefeld 2004; Liu/Johnson 2005; Jiménez/San Martín 2010). Nach Swaminathan et al. (2007, 248ff.) sowie Fetscherin und Toncar (2010, 175ff.) wurde bis dato der COO-Effekt auf die zentralen Konstrukte von Marken-Konsumenten-Beziehungen, insbesondere die Markenzufriedenheit, das Markencommitment und das Markenvertrauen gänzlich vernachlässigt. Zudem bleibt bislang die Frage unbeantwortet, welche Massnahmen von Unternehmen einzusetzen sind, damit ein COO die von Konsumenten wahrgenommene Markenpersönlichkeit positiv beeinflusst (u. a. Heimbach et al. 1989; Tse/Gorn 1993; Jiménez/San Martín 2010). Obwohl die Schweiz international angesehen ist und mehrheitlich mit positiven Eigenschaften in Verbindung gebracht wird, existieren bislang nur vereinzelt Studien, die die Schweiz als COO betrachten (Li/Wyer Jr. 1994; Kühn/ Weiss 1998; Feige et al. 2008; Feige et al. 2010). Ein letzter Kritikpunkt an existierenden Studien zum COO ist die häufige Wahl von Studenten-Samples, die nach Verlegh und Steenkamp $(1999,537)$ sowie Peterson $(2001,450 \mathrm{ff}$.$) eine höhere Homogenität des Ant-$ wortverhaltens aufweisen.

In Anbetracht der aufgezeigten Forschungslücken werden die folgenden Zielsetzungen in der vorliegenden Studie verfolgt: Zum einen gilt es, die Wirkungsbeziehungen des COO auf psychologische und verhaltensbezogene Grössen, insbesondere die zentralen Konstrukte der Marken-Konsumenten-Beziehung - Markenzufriedenheit, Markencommitment sowie Markenvertrauen - und die Markenbindung, zu analysieren. Zum anderen setzt sich die vorliegende Studie zum Ziel, ein Modell zu entwickeln, das Rückschlüsse auf die Qualität der kommunikativen Übertragung des COO zulässt. Dabei wird die Schweiz als COO herangezogen und auf eine repräsentative Stichprobe von Konsumenten zurückgegriffen. 


\begin{tabular}{|c|c|c|c|c|c|c|c|c|c|}
\hline 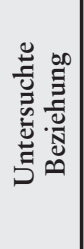 & 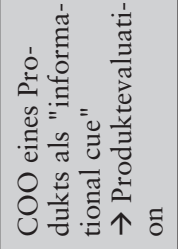 & 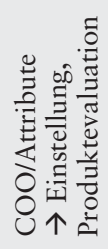 & 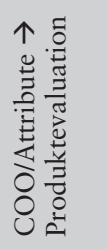 & 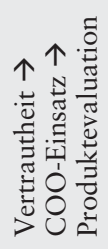 & 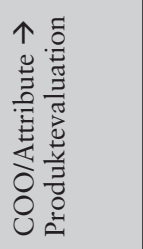 & 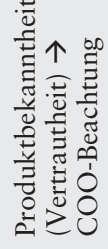 & 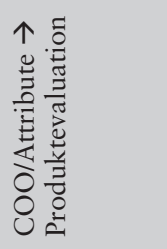 & 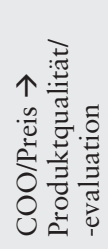 & 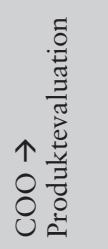 \\
\hline 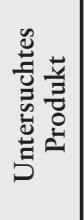 & 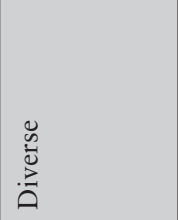 & 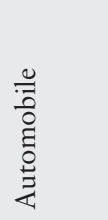 & 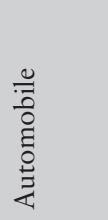 & 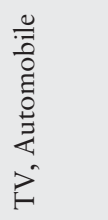 & 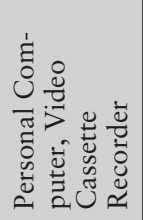 & $\begin{array}{l}\frac{0}{30} \\
0 \\
\vdots \\
0 \\
0 \\
0\end{array}$ & 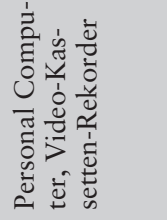 & 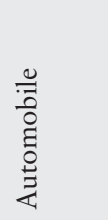 & 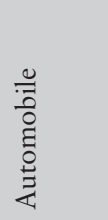 \\
\hline 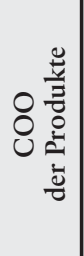 & 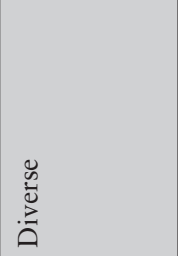 & 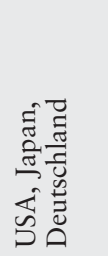 & 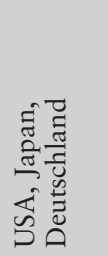 & 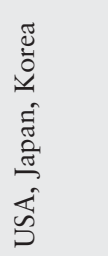 & 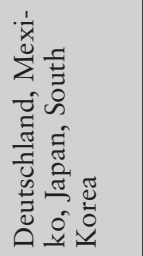 & 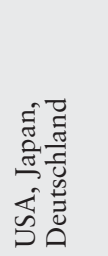 & 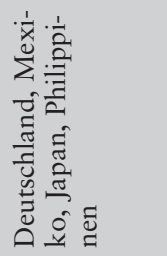 & 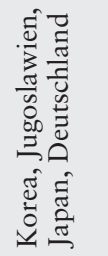 & 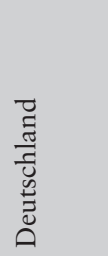 \\
\hline 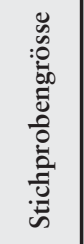 & 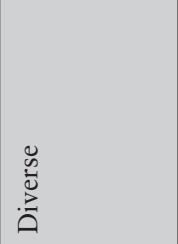 & 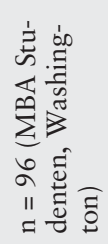 & 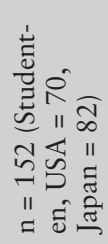 & $\begin{array}{l}0 \\
= \\
\text { II } \\
=\end{array}$ & 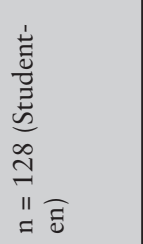 & 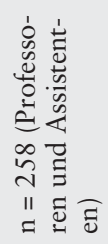 & 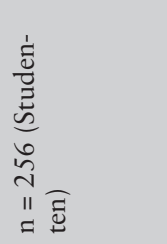 & 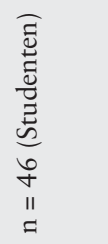 & 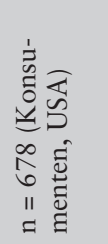 \\
\hline 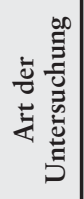 & 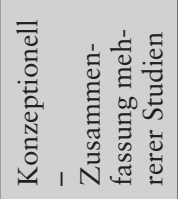 & 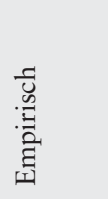 & 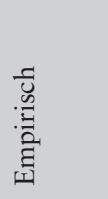 & 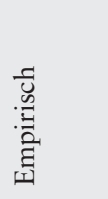 & 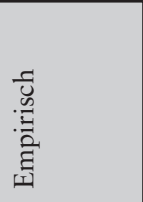 & 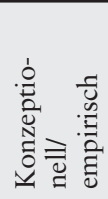 & 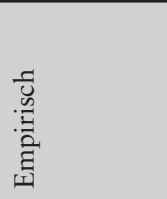 & 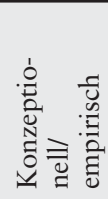 & 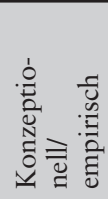 \\
\hline$\stackrel{\Xi}{=}$ & 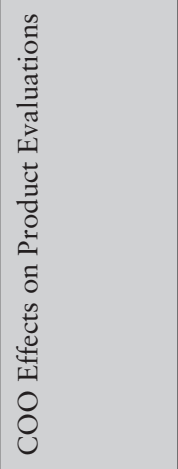 & 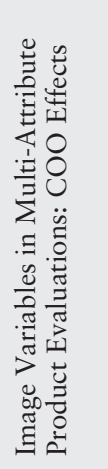 & 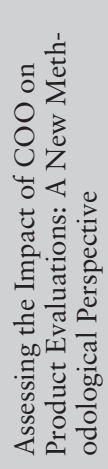 & 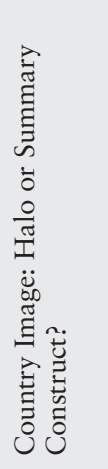 & 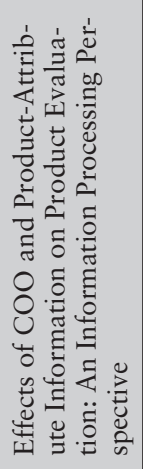 & 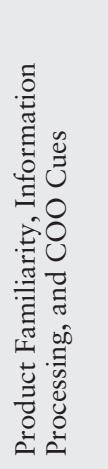 & 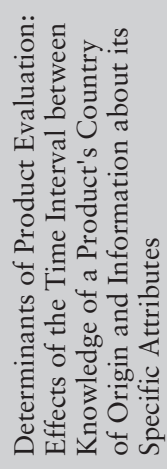 & 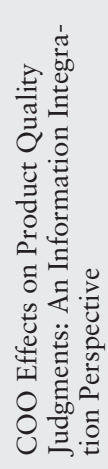 & 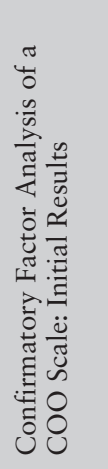 \\
\hline 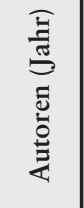 & 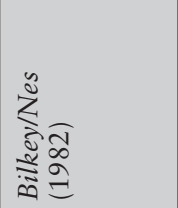 & 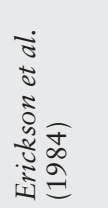 & 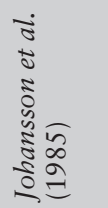 & $\begin{array}{l}\widehat{\sigma} \\
\infty \\
0 \\
= \\
\vdots \\
\vdots \\
\vdots\end{array}$ & 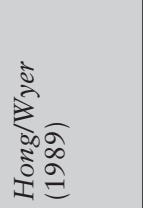 & 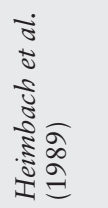 & 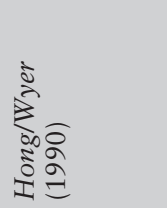 & 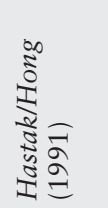 & 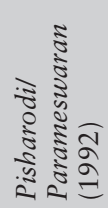 \\
\hline
\end{tabular}




\begin{tabular}{|c|c|c|c|c|c|c|c|c|c|}
\hline 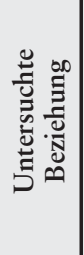 & 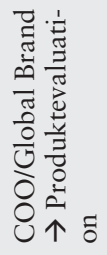 & 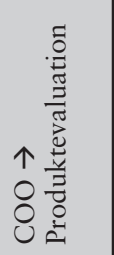 & 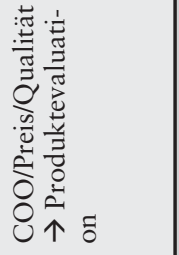 & 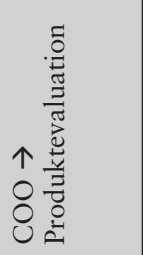 & 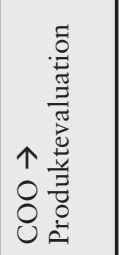 & 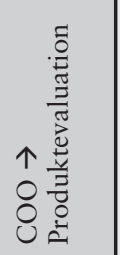 & 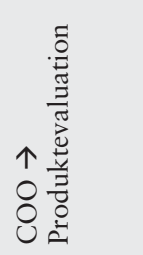 & 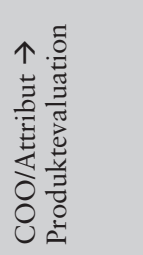 & 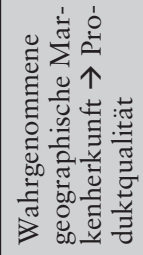 \\
\hline 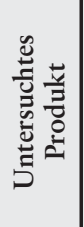 & 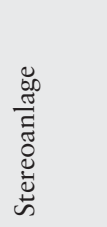 & & 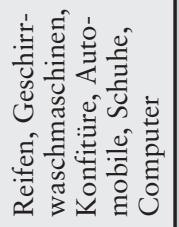 & 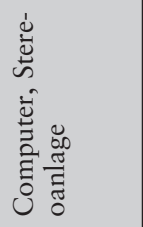 & 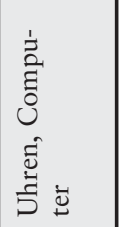 & ' & 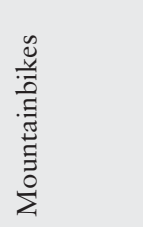 & 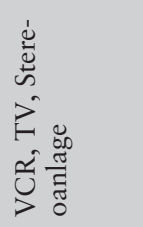 & 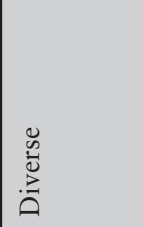 \\
\hline 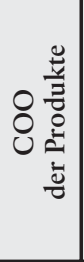 & 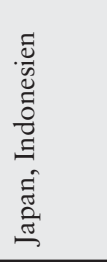 & 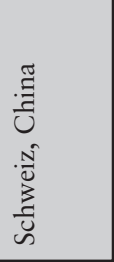 & 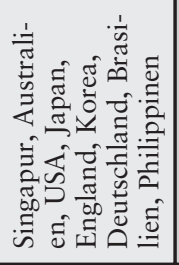 & 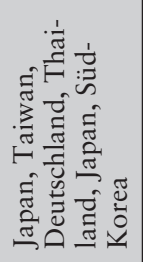 & 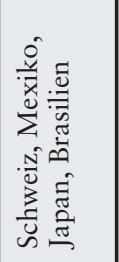 & ' & 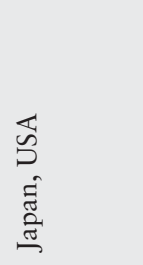 & 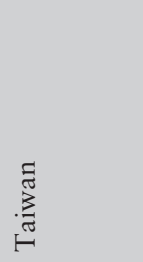 & 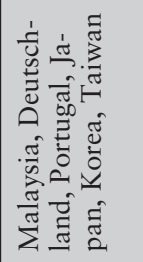 \\
\hline 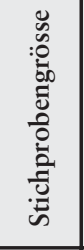 & 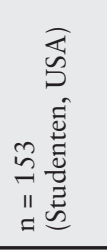 & 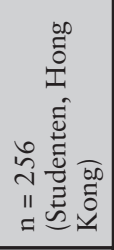 & 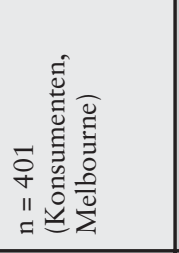 & 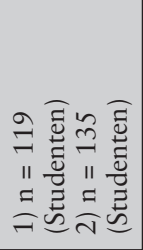 & 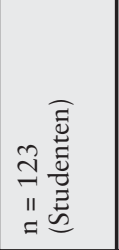 & ' & 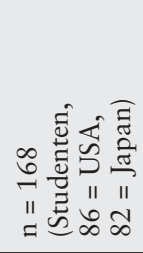 & 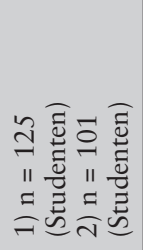 & 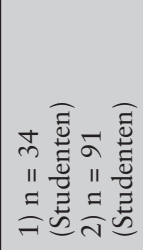 \\
\hline 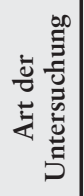 & 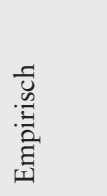 & 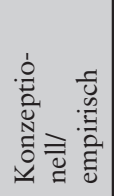 & 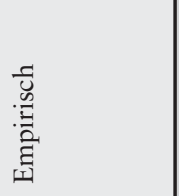 & 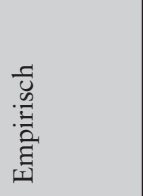 & 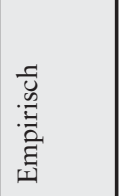 & 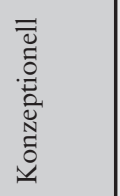 & 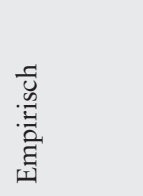 & 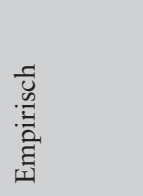 & 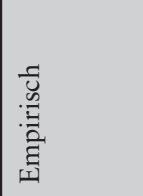 \\
\hline 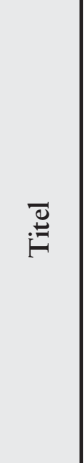 & 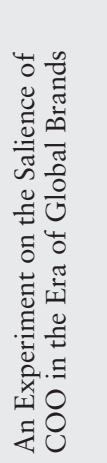 & 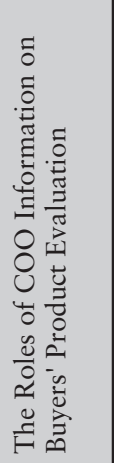 & 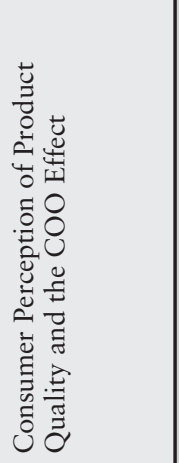 & 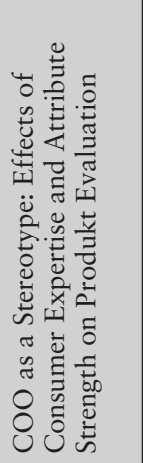 & 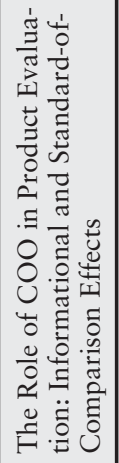 & 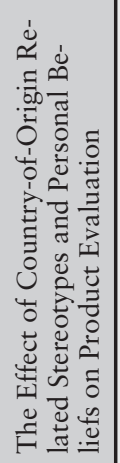 & 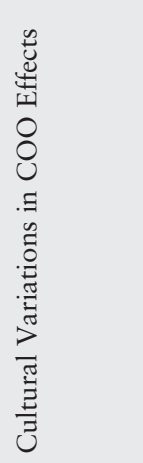 & 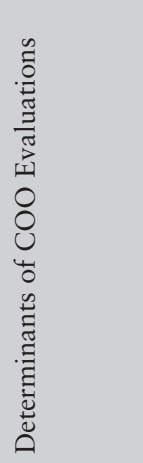 & 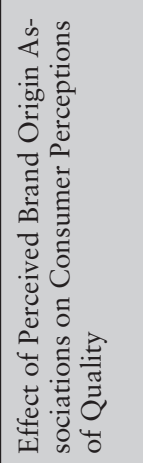 \\
\hline 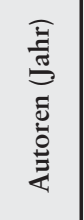 & 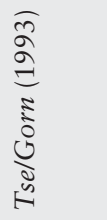 & 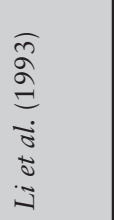 & 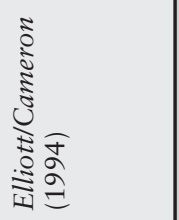 & 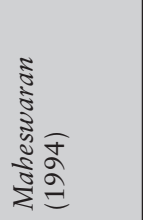 & $\frac{\overline{+}}{2}$ & 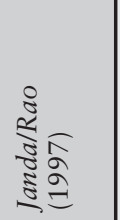 & 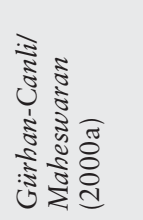 & 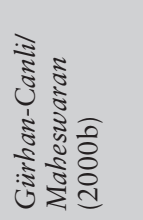 & 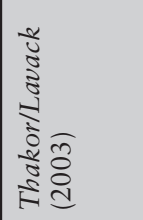 \\
\hline
\end{tabular}




\begin{tabular}{|c|c|c|c|c|c|c|c|}
\hline 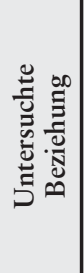 & 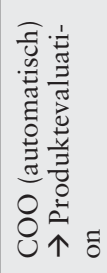 & 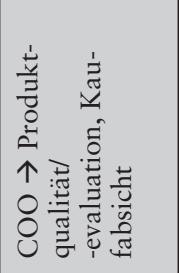 & 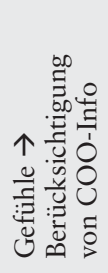 & 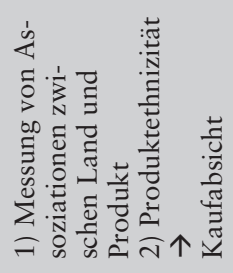 & 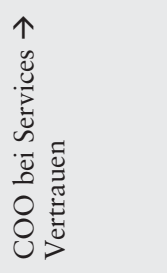 & 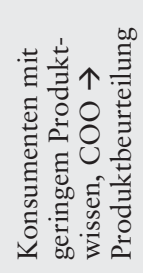 & 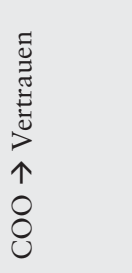 \\
\hline 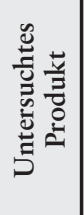 & 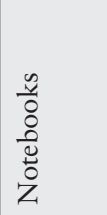 & 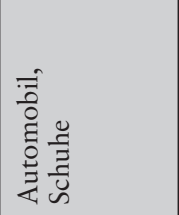 & 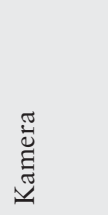 & 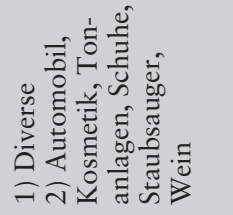 & 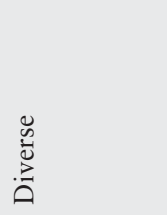 & 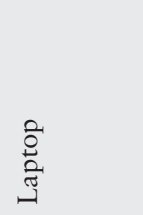 & 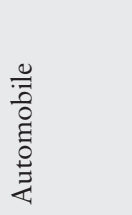 \\
\hline 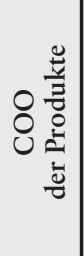 & 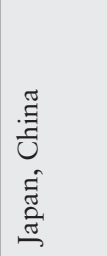 & 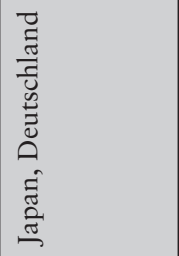 & 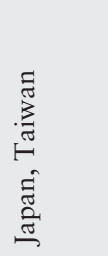 & 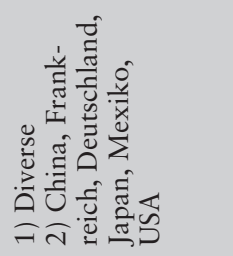 & $\frac{\tilde{0}}{0}$ & 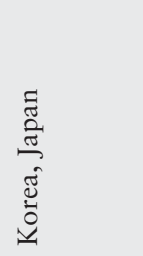 & 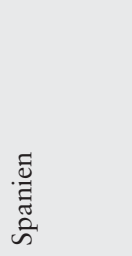 \\
\hline 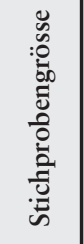 & 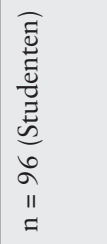 & 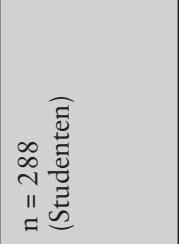 & 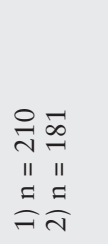 & 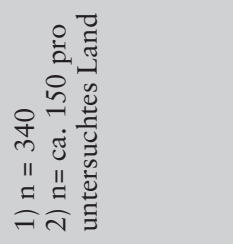 & 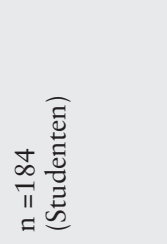 & 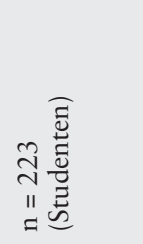 & 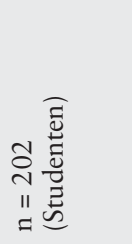 \\
\hline 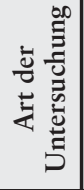 & 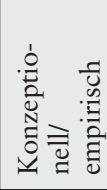 & 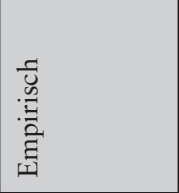 & 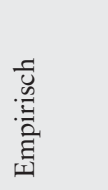 & 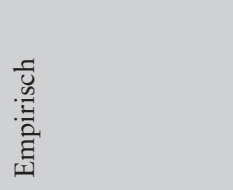 & 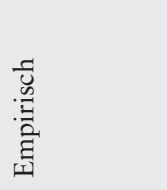 & 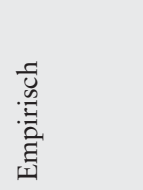 & 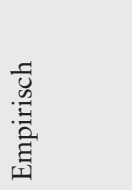 \\
\hline$\stackrel{\Xi}{\Xi}$ & 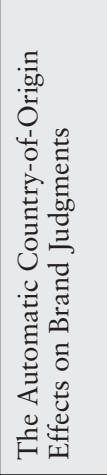 & 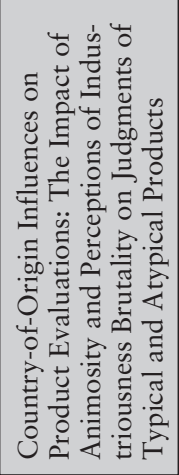 & 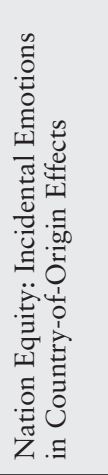 & 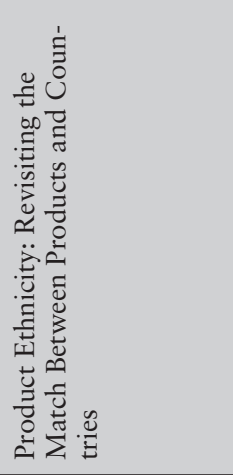 & 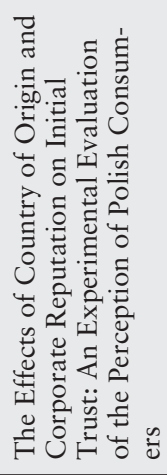 & 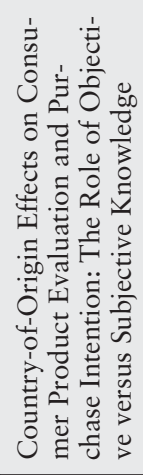 & 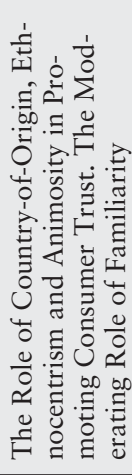 \\
\hline 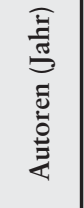 & 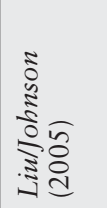 & 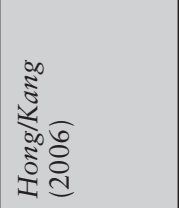 & 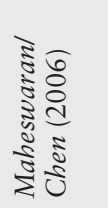 & 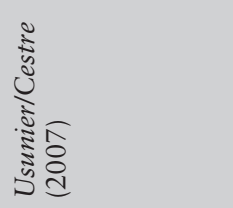 & 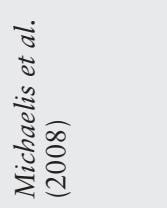 & 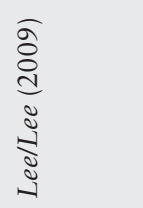 & 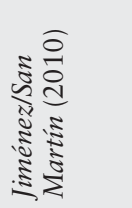 \\
\hline
\end{tabular}

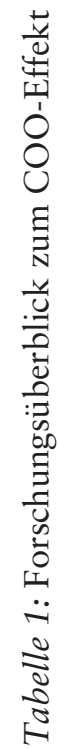


Zusammenfassend wird mittels Kausalanalyse am Beispiel der Marke Ricola die Übermittlung der Schweiz als COO (Swissness) überprüft und der Einfluss der Swissness auf die wesentlichen Grössen der Marken-Konsumenten-Beziehung sowie die Markenbindung verifiziert. Aus den Ergebnissen lassen sich Implikationen für das Management einer internationalen Markenführung an einem konkreten Beispiel ableiten.

\section{Theoretischer Bezugsrahmen}

Die Analyse des Einflusses des COO-Effekts auf Marken-Konsumenten-Beziehungen und das Konsumentenverhalten bedingt die theoretische Fundierung von zwei Fragestellungen. Zum einen gilt es die Frage zu beantworten, warum der COO die konsumentenseitige Wahrnehmung einer Marke beeinflusst und zum anderen ist zu begründen, wie der Einfluss des $\mathrm{COO}$ auf die Marken-Konsumenten-Beziehungen entsteht.

Im Rahmen eines Kaufentscheidungsprozesses wird die konsumentenseitige Wahrnehmung einer Marke vom COO beeinflusst (Cordell 1992, 251ff.). Die Überlegungen zum Einfluss des COO-Effekts auf die Wahrnehmung einer Marke haben ihren Ursprung in der Animismustheorie. Diese geht davon aus, dass Individuen dazu neigen, nicht-lebende Objekte, wie z. B. passive Marken, zu vermenschlichen, um durch die Schaffung einer Markenpersönlichkeit Interaktionen mit der Marke zu vereinfachen (u. a. Aaker 1997; Fournier 1998; Baner et al. 2002). Nach der Animismustheorie erfolgt die Vitalisierung einer Marke in der Markenkommunikation meist durch den Einsatz von Überträgern, wie z. B. dem COO. Dabei wird durch die Übertragung von Eigenschaften des COO auf eine Marke aus Konsumentensicht eine Markenpersönlichkeit geschaffen (Fournier 1998, 343f.). Die einzelnen Marketingmassnahmen in Verbindung mit dem COO werden vom Konsumenten als Handlungen der Marke interpretiert und daraus Rückschlüsse auf die Markenpersönlichkeit gezogen (Fournier 1998, 348). Als Grundvoraussetzung für eine günstige Beeinflussung der wahrgenommenen Markenpersönlichkeit ist die positive Wahrnehmung des Herkunftslandes zu nennen. Nur wenn ein Herkunftsland ein positives Image besitzt, ist ein Imagetransfer auf die Markenpersönlichkeit für Unternehmen interessant (Bilkey/Nes 1982, 89ff.; Kühn 1992; Li et al. 1993).

Im Folgenden ist der Einfluss des COO auf die Marken-Konsumenten-Beziehung theoretisch zu fundieren. Einen Erkenntnisgewinn liefert in diesem Zusammenhang die Theorie der sozialen Durchdringung, die im Ursprung zur Begründung der Entwicklung von zwischenmenschlichen Beziehungen herangezogen wurde (Altman/Taylor 1973). Diese gibt ebenfalls Aufschluss über die Entstehung, Weiterentwicklung und Beendigung von Marken-Konsumenten-Beziehungen und steht dabei nicht in konkurrierender Beziehung zur Animismustheorie sondern wird vielmehr von dieser ergänzt (Taylor et al. 2002). Nach der Theorie der sozialen Durchdringung entdecken Individuen durch Interaktionen mit einer Marke im Laufe der Zeit weitere Facetten der Markenpersönlichkeit und entwickeln eine Beziehung von anfangs oberflächlichen zu immer tieferen, intimeren Formen (Veloutsou 2007, 17ff.). Die über Marketingmassnahmen vermittelten Informationen über den $\mathrm{COO}$ werden, wie in der Animismustheorie erläutert, vom Konsumenten als eine Facette der Markenpersönlichkeit wahrgenommen (Fournier 1998, 348f.). In Anlehnung an Iacobucci und Hibbard (1999, 15), Bengtsson (2003, 154f.) und Veloutsou (2007, 15) wirkt der COO-Effekt über wechselseitige Interaktionen und die damit wahrgenommene Markenpersönlichkeit auf die Entstehung von Marken-Konsumenten-Beziehungen. Die Beziehungsintensität zwischen Konsument und Marke wird dabei anhand der beiden Di- 
mensionen Persönlichkeitsbreite und -tiefe bestimmt. Im Rahmen der Persönlichkeitsbreite wird festgehalten, wie viele unterschiedliche Kategorien der Persönlichkeit einer Marke dem Gegenüber eröffnet und wie häufig diese thematisiert werden. Die Persönlichkeitstiefe beinhaltet dagegen, inwieweit eine Marken-Konsumenten-Beziehung entweder oberflächlich bleibt oder zentrale Merkmale einer Markenpersönlichkeit offenbart. Nach jeder Interaktion mit einer Marke werden die Interaktionsergebnisse von Konsumenten bewertet und die Intensität der Marken-Konsumenten-Beziehung entsprechend beeinflusst (Grönroos 2000, 5ff.).

Die Animismustheorie und die Theorie der sozialen Durchdringung liefern demnach wichtige Erkenntnisbeiträge für den Einfluss des $\mathrm{COO}$ auf die wahrgenommene Markenpersönlichkeit und die Entstehung von Marken-Konsumenten-Beziehungen (Taylor et al. 2002, 270ff.). Der Beziehungsaufbau vollzieht sich durch wiederholte Interaktionen, in denen es zu einem Informationsaustausch kommt und das Ausmass der konsumentenseitigen Kenntnisse über einzelne Facetten der Markenpersönlichkeit steigt (Grönroos 2000, 11). Neben der Entstehung von Marken-Konsumenten-Beziehungen lassen sich daraus auch Erkenntnisse zur Beziehungsintensität ableiten. Die Animismustheorie sowie die Theorie der sozialen Durchdringung stellen somit den theoretischen Ordnungsrahmen der vorliegenden Arbeit dar.

\section{Modellaufbau und Ableitung von Hypothesen}

\subsection{Swissnessquantität und -qualität als Determinanten}

Die Voraussetzung für einen steigenden Einfluss des Herkunftslandes Schweiz auf Kaufentscheidungen ist eine positive kundenseitige Wahrnehmung der Schweiz. Nur wenn die Schweiz über ein positives Image verfügt, ist ein Imagetransfer für Unternehmen erstrebenswert (Bilkey/Nes 1982, 89ff.). Die Präferenz für ein Land entsteht ausschliesslich in der Wahrnehmung des Landes durch den Konsumenten. Diese wird auf Basis eigener Erfahrungen, z. B. mit Einwohnern und Produkten oder durch politische und ökonomische Berichte sowie Erfahrungen von Dritten gebildet und entsteht in einem langfristigen Entwicklungsprozess (Kühn/Weiss 1998). Aufgrund des langen Entwicklungsprozesses ist das Landesimage bei Konsumenten stark verfestigt und somit Änderungen gegenüber relativ resistent (Kühn 1992). Für das Markenmanagement stellt demnach das COO eine solide Positionierungsbasis dar. Durch die Verknüpfung einer Marke mit dessen COO bieten Unternehmen ihren Konsumenten eine Orientierungshilfe sowie Sicherheit und unterstützen die Entstehung von Marken-Konsumenten-Beziehungen. Die Schweiz erfüllt als COO die o.g. Anforderung, gilt weltweit als politisch stabiles Land mit einer hohen Lebensqualität und wird von Konsumenten meist als attraktiv, zuverlässig und vertrauenswürdig wahrgenommen. Laut einer Studie stehen Schweizer Marken für Qualität, Zuverlässigkeit und Exklusivität (Feige et al. 2008, 14ff.). Die Schweiz wird von Schweizer Unternehmen vermehrt zur Unterstützung des Erfolgs lokal ansässiger Marken herangezogen. Ebenso, wie die Schweiz in der Kommunikation von Schweizer Unternehmen als „Gütesiegel“ eingesetzt wird, weisen auch andere Herkunftsländer mit einem positiven Image ein erhebliches Erfolgspotenzial für Unternehmen auf (Feige et al. 2008, 11). Für Markenanbieter stellt sich die Herausforderung, die positiven Imagewerte des COO auf eine gewünschte Marke zu übertragen und im Rahmen der Kommunikation diese Werte möglichst effektiv zu übermitteln. 
Die Swissness wird im vorliegenden Artikel wie folgt definiert: „Swissness ist ein latentes Konstrukt, das alle von Konsumenten wahrgenommene Eigenschaften der Schweiz als Herkunftsland, deren Produkte sowie Unternehmen umfasst und durch Übertragung von Schweiz-typischen Symbolen und Merkmalen in der Marketing- und Unternehmenskommunikation aktiviert wird.“

Die Ableitung spezifischer Aussagen zu unternehmensseitigen COO bezogenen Kommunikationsmassnahmen erfordert, aufbauend auf dieser Definition, eine Spezifizierung der Swissness. Der Animismustheorie folgend sind Unternehmen in der Lage, die wahrgenommene Markenpersönlichkeit Schweizer Produkte durch die Schweiz als Herkunftsland bzw. die Swissness zu beeinflussen (Fournier 1998). In Anlehnung an die Theorie der sozialen Durchdringung wird die Beziehungsintensität zwischen einer Marke und Konsumenten von den Dimensionen Persönlichkeitsbreite und -tiefe bestimmt (Taylor et al. 2002, 271f.). Demzufolge sind im Rahmen der Interaktionen eines Konsumenten mit einer Marke, die beiden Dimensionen der Markenpersönlichkeit, Persönlichkeitsbreite und -tiefe für den Aufbau von Marken-Konsumenten-Beziehungen entscheidend. Die Persönlichkeitsbreite umfasst mit der Anzahl unterschiedlicher Merkmale der Markenpersönlichkeit, die Konsumenten entdecken und der Häufigkeit, mit der diese wahrgenommen werden eher quantitative Aspekte. Im Gegensatz dazu beinhaltet die Persönlichkeitstiefe mit der Berücksichtigung zentraler tiefer gehender Merkmale der Markenpersönlichkeit eher qualitative Aspekte (Grönroos 2000, 6ff.). Der Einfluss der Swissness auf die wahrgenommene Markenpersönlichkeit ist nach Romaniuk und Sharp (2003, 219ff) mit der von Konsumenten wahrgenommenen Kommunikation gleichzusetzen und wird als die konsumentenseitige Bewertung der Quantität und Qualität von über die Schweiz vermittelten Kommunikationsbotschaften determiniert. Aufbauend auf diesen Ausführungen werden im Rahmen der vorliegenden Studie aus der Swissness die beiden Konstrukte Swissnessquantität und Swissnessqualität abgeleitet. Swissnessquantität umfasst dabei den Druck bzw. die Präsenz, mit der kommunikative Massnahmen umgesetzt werden und ergibt sich aus der Häufigkeit bzw. Intensität, mit der kommunikative Massnahmen mit der Schweiz als COO einer Marke wiederholt werden (Fang et al. 2007, 98ff.). Für die Swissnessquantität wird folgende Definition dargelegt: „Swissnessquantität ist ein latentes Konstrukt, das die Dauer und Häufigkeit der Markenunterstützung durch die Swissness umfasst und wird durch die Einbindung von Swissness-Elementen in den Marketingmix gesteuert."

Nach Kühn und Weiss (1998, 50ff.) sowie Chen et al. (2007, 1047f.) ist bei der Übertragung von Imagewerten eines COO auf eine Marke vor allem die Qualität der kommunikativen Vermittlung COO spezifischer Informationen entscheidend. Nur wenn Konsumenten das $\mathrm{COO}$ im Rahmen der Markenkommunikation wiedererkennen, findet eine positive Beeinflussung der Markenpersönlichkeit statt (Chen et al. 2007, 1049f.). Samiee et al. (2005) prägten im Hinblick auf die Fähigkeit von Konsumenten, das Herkunftsland einer Marke wiederzuerkennen, den Begriff „Brand Origin Recognition Accuracy (BORA)“. Zudem hängt die Übertragung des positiven Landesimages auf ein Unternehmen oder dessen Marken von der Identifikation des Unternehmens mit dem Herkunftsland ab (Kühn/ Weiss 1998, 51ff.). Fleck und Quester (2007, 976ff.) sprechen in diesem Zusammenhang von der Kongruenz zwischen den relevanten Charakteristika einer Marke und dem COO als „Match-Objekt“. Hierbei wird der kognitive Transferprozess von Imagewerten der Schweiz zu spezifischen Marken bei Konsumenten durch die Übereinstimmung wesentlicher Merkmale der beiden Objekte unterstützt (Fleck/Quester, 2007, 977f.). Dementspre- 
chend umfasst die Swissnessqualität die wahrgenommene Kongruenz zwischen COO und Marke sowie die Qualität der kommunikativen Übermittlung spezifischer COO-Informationen und wird in dem vorliegenden Beitrag wie folgt definiert:

Swissnessqualität ist ein latentes Konstrukt, das die vom Konsumenten wahrgenommene Kongruenz zwischen Merkmalen der Produkt- bzw. Unternehmensmarke und den Attributen der Schweiz als COO sowie die die Qualität der kommunikativen Übermittlung der spezifischen COO-Informationen umfasst.

In der vorliegenden Studie werden die Swissnessquantität und Swissnessqualität als Massnahmen des Unternehmens in Bezug auf die Schweiz als COO erfasst. Anschliessend sind die Wirkungen der Swissnessquantität und Swissnessqualität auf die wesentlichen Konstrukte von Marken-Konsumenten-Beziehungen - Markenzufriedenheit, Markencommitment und Markenvertrauen - zu untersuchen. Letztlich wird der Einfluss der Konstrukte der Marken-Konsumenten-Beziehung auf die Markenbindung betrachtet. In Abbildung 1 wird das entsprechende Untersuchungsmodell dargestellt.

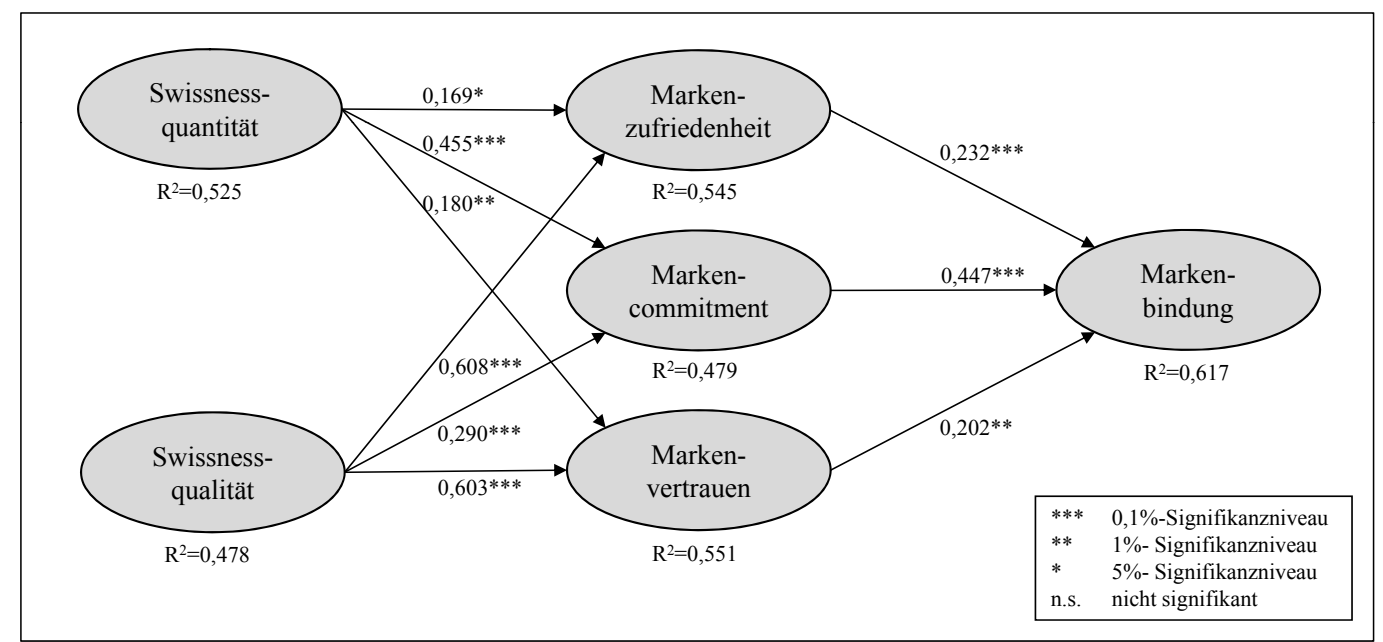

Abbildung 1: Modell zur Messung der Swissness mit Hypothesensystem

\subsection{Konzeptualisierung der Markenbeziehung}

Die Identifikation der wichtigsten Grössen von Marken-Konsumenten-Beziehungen wurde in einer Vielzahl von Studien empirisch nachgewiesen. In Anlehnung an u. a. Walter et al. (2003) sowie Farrelly und Quester (2005) werden Marken-Konsumenten-Beziehungen über die zentralen Konstrukte Markenzufriedenheit, Markencommitment und Markenvertrauen gemessen. In den nachstehenden Abschnitten werden diese drei Konstrukte näher betrachtet.

Markenzufriedenheit resultiert aus einem Soll-Ist-Vergleich zwischen den Erwartungen eines Individuums und den tatsächlich von der Marke erbrachten Leistungen. Entspricht die wahrgenommene Leistung den Erwartungen oder übertrifft sie diese, entsteht Zufriedenheit. Liegt die Ist-Leistung hingegen unter der Soll-Leistung, führt dies zu Unzufriedenheit (Churchill/Surprenant 1982, 493ff.). In Austauschbeziehungen, wie beispielsweise Marken-Konsumenten-Beziehungen, stellt die Markenzufriedenheit eine zentrale Zielgrös- 
se dar. Esch et al. $(2006,100)$ konstatieren, dass „the primary positive outcome of an exchange relationship is satisfaction “. Da Austauschbeziehungen primär auf einem KostenNutzen-Kalkül basieren, erwarten Konsumenten, dass Leistungen und Gegenleistungen in einem ausgeglichenen Verhältnis zueinander stehen. Oliver und DeSarbo $(1988,504)$ stellen in diesem Zusammenhang fest: ,[...] parties to an exchange will feel equitably treated and thus satisfied if the ratio of their outcomes to inputs is in some sense fair".

Unter Markencommitment wird die gefühlsmässige Bindung des Konsumenten zur Marke verstanden. Demnach werden sozial motivierte Marken-Konsumenten-Beziehungen nicht vom Bedürfnis nach Gerechtigkeit sondern durch Gefühle, die mit der Beziehung verbunden sind, beherrscht (Delgado-Ballester/Munuera-Alemán 2001). Die Bedeutung von Commitment für den Aufbau und Erhalt langfristiger Marken-Konsumenten-Beziehungen ergibt sich analog zu nahen zwischenmenschlichen Beziehungen aus dem psychischen Wohlbefinden, den intime, emotionale Markenbeziehungen dem Nachfrager stiften. Nahe Beziehungen geben den Beziehungspartnern u. a. das Gefühl der emotionalen Sicherheit und Stabilität (Chaudhuri/Holbrook 2002).

Letztlich ist das Markenvertrauen als Erfolgsgrösse sozial geprägter Marken-Konsumenten-Beziehungen anzusehen. Markenvertrauen ergibt sich in erster Linie aus der Komplexitäts- und Unsicherheitsreduktion, die mit dem Vertrauen in eine Marke einhergehen und in der Folge die Interaktionseffizienz erhöhen (Casalo et al. 2008, 23). Dabei ist das Markenvertrauen dadurch gekennzeichnet, dass sich der Konsument auf wahrgenommene Markeneigenschaften verlässt und dadurch das wahrgenommene Kaufrisiko sowie Kosten und Mühen, die mit einem Kauf verbunden sind, reduziert. Sofern diese Erwartung von einer Marke erfüllt wird, kommt es zu einer Festigung des Vertrauens und der Marken-Konsumenten-Beziehung (Walter et al. 2003).

Wie in Abbildung 1 dargestellt, werden im vorliegenden Beitrag Wirkungsbeziehungen von der Swissnessquantität und Swissnessqualität auf die drei Konstrukte der MarkenKonsumenten-Beziehung vermutet. Um die Wirkung der Swissnessquantität auf die Konstrukte Markenzufriedenheit, Markencommitment und Markenvertrauen herzuleiten, wird der Mere-Exposure-Effekt herangezogen. Der Effekt zeigt, dass: „mere repeated exposure of the individual to a stimulus is a sufficient condition for the enhancement of his attitude toward it. By ,mere exposure" is meant a condition which just makes the given stimulus accessible to the individuals perception " (Zajonc 1968, 1). Der Mere-Exposure-Effekt gehört den automatischen Lernprozessen an, d. h. Aufnahme und Speicherung von Informationen erfolgen unbewusst und erfordern kein anspruchsvolles Lernmuster. Die Kommunikationsmassnahmen der Swissness werden unbewusst wahrgenommen und der Konsument bildet durch häufige Kontakte Präferenzen zum Werbeobjekt, wie z. B. zur Unternehmensoder Produktmarke. Die zunehmende Geläufigkeit des Stimulus resultiert in Vertrautheit und einer besseren Einordnung des Werbeobjekts (Fang et al. 2007, 97). Nach dem MereExposure Effekt resultiert der vermehrte Kontakt mit dem Stimulus Swissness in einer positiven affektiven Reaktion und einer positiven Einstellung, die wiederum auf die Konstrukte Markenzufriedenheit, Markencommitment und Markenvertrauen wirkt (Esch et al. 2006, 100ff.). Demnach gilt es, folgende Hypothesen zu überprüfen:

H1: Die Swissnessquantität hat einen positiven Einfluss auf die Markenzufriedenheit.

H2: Die Swissnessquantität hat einen positiven Einfluss auf das Markencommitment.

H3: Die Swissnessquantität hat einen positiven Einfluss auf das Markenvertrauen. 
Der Einfluss zwischen der Swissnessqualität und den Konstrukten der Marken-Konsumenten-Beziehung wird theoretisch über die Match-up-Hypothese begründet (Fleck/Quester 2007, 981). In einer Studie von Roth und Romeo (1992) wurde die Match-up-Hypothese erstmals zur Wirkungsanalyse im Zusammenhang mit der Kongruenz zwischen COO und Marken eingesetzt. Dabei wurde der „Fit“ zwischen dem Herkunftsland und einer Produktkategorie untersucht und bei hoher Übereinstimmung eine positivere Wahrnehmung der Marke sowie verstärkende Wirkung des COO auf das Kaufverhalten nachgewiesen (Roth/Romeo 1992, 493). Die Match-up-Hypothese geht davon aus, dass „endorsers are more effective when there is a ,fit' between the endorser and the endorsed product" (Till) Busler 2000, 1f.). Weitere Forschungsergebnisse von Fleck und Quester (2007, 993) zeigen, dass im Bereich der Kommunikation die Übereinstimmung zwischen Marke und Match-Objekt einen positiven Einfluss auf die wahrgenommene Kommunikation und die Markeneinstellung von Konsumenten hat. Bei Übertragung auf die Swissness ist davon auszugehen, dass der Effekt der Swissnessqualität auf Marken-Konsumenten-Beziehung höher ist, wenn eine Übereinstimmung bzw. ein „Fit“ zwischen dem Herkunftsland und der Marke besteht. Somit werden die folgenden Hypothesen aufgestellt:

H4: Je höher die Swissnessqualität ist, desto höher ist die Markenzufriedenheit.

H5: Je höher die Swissnessqualität ist, desto grösser ist das Markencommitment.

H6: Je höher die Swissnessqualität ist, desto grösser ist das Markenvertrauen.

\subsection{Markenbindung als Konsequenz von Markenbeziehungen}

Die Markenzufriedenheit stellt eine zentrale Einflussgrösse der Markenbindung dar (u. a. De Wulf et al. 2001; Aaker et al. 2004). Eine theoretische Erklärungsperspektive für die Bedeutung der Markenzufriedenheit liefert das Confirmation/Disconfirmation-Paradigma (Homburg et al. 1999). Mit dem Kauf einer Marke verbinden Konsumenten bestimmte subjektive Markenerwartungen, die mit den tatsächlichen Erfahrungen mit der Marke verglichen werden. Der Vergleich zwischen Anspruchsniveau und Wahrnehmung durch den Kunden führt zu einer Übererfüllung (positive Diskonfirmation), Erfüllung (Konfirmation) oder Untererfüllung (negative Diskonfirmation) der Kundenerwartungen, die sich in Markenzufriedenheit oder -unzufriedenheit ausdrücken (Homburg et al. 1999). Das Empfinden von Gerechtigkeit bzw. Zufriedenheit hat wiederum eine stabilisierende Wirkung auf die Markenbindung. Empfindet der Kunde hingegen die Verteilung als ungerecht, so kommt es zu inneren Spannungen bzw. zu Unzufriedenheit (Gierl/Bartikowski 2003, 14ff.). Somit wird die folgende Hypothese aufgestellt:

H7: Je höher die Markenzufriedenheit eines Konsumenten ist, desto eher entwickelt sich eine intensive Markenbindung.

Das Markencommitment wird als zweites Konstrukt der Markenbindung herangezogen und als die emotionale Bindung des Kunden zur Marke verstanden. Beim Vorliegen von Markencommitment entsteht bei Konsumenten eine hohe Identifikation mit der Marke sowie ein Gefühl der inneren Verpflichtung und Verbundenheit (Morgan/Hunt 1994, 20ff.). Ein gesteigertes Markencommitment ist der Ausdruck einer erfolgreichen Marken-Konsumenten-Beziehung und verstärkt den Wunsch des Konsumenten, die Beziehung zur Marke aufrecht zu erhalten (Delgado-Ballester/Munuera-Alemán 2001, 1240). In einer empirischen Studie von Garbarino und Johnson (1999, 71) wird das Markencommitment als 
wichtiger Faktor von langfristigen Beziehungen identifiziert. Daraus folgt die nachstehende Hypothesenformulierung:

H8: Je höher das Markencommitment eines Konsumenten ist, desto eher entwickelt sich eine intensive Markenbindung.

Das Markenvertrauen wird als Einflussfaktor der Markenbindung aus der Komplexitäts- und Unsicherheitsreduktion abgeleitet, die mit dem Vertrauen in eine Marke einhergehen (Casalo et al. 2008, 23). Als theoretische Begründung ist die Risikotheorie heranzuziehen, nach der Konsumenten bestrebt sind, das Risiko in Kaufsituationen zu minimieren. Dementsprechend werden Marken bevorzugt, zu denen sie auf Basis bisheriger Markenerfahrungen Vertrauen aufgebaut haben. Die Höhe des wahrgenommenen Kaufrisikos wird wiederum vom Ausmass der Unsicherheit und der Erheblichkeit der Konsequenzen eines Fehlkaufs bestimmt. „[...] most disciplines agree that risk is a critical condition for trust to influence choice and behavior" (Delgado-Ballester et al. 2003, 37). Folglich hat Markenvertrauen eine stabilisierende Wirkung auf die Markenbindung (De Wulf et al. 2001). Delgado-Ballester und Munuera-Aleman (2001, 1240) beschreiben Markenvertrauen als eine,$[. .$.$] key variable in the development of an enduring desire to maintain a relationship$ in the long term, for example with a brand“. Daraus wird folgende Hypothese abgeleitet:

H9: Je höher das Markenvertrauen eines Konsumenten ist, desto eher entwickelt sich eine intensive Markenbindung.

\section{Konzeption und Ergebnisse der empirischen Untersuchung}

\subsection{Design und Durchführung der empirischen Untersuchung}

Die Überprüfung des Hypothesensystems wird auf Basis einer empirischen Untersuchung vorgenommen. Die hierfür erforderlichen Daten wurden im Rahmen einer anonymen Online-Befragung unter deutschen Konsumenten erhoben. Die Befragung von „Nicht-Schweizern" scheint im Zusammenhang mit der Untersuchung der Swissness als vorteilhaft, da somit eine Überbewertung des COO vermieden wird. Aufgrund der hohen internationalen Bekanntheit und der Unterstützung der Marke durch typische Swissnesselemente wurde die Marke Ricola als geeignetes Untersuchungsobjekt der Konsumgüterbranche identifiziert.

Der für die Erhebung entwickelte Fragebogen umfasst insgesamt 32 Fragen, wobei fünf zur Erfassung von soziodemografischen Daten dienen und die übrigen auf die Überprüfung des Modells abzielen. Die Probanden wurden dazu aufgefordert, anhand einer 7-stufigen Likertskala den Grad ihrer Zustimmung bzw. Ablehnung anzugeben. Hierbei wurden jeweils die Extrempunkte 1 mit „stimme überhaupt nicht zu“ und 7 mit „stimme voll und ganz zu" verbalisiert. Per E-Mail wurden 2.500 Probanden zur Mitwirkung an der Studie gebeten. Mit einer Rücklaufquote von 12,48 Prozent beteiligten sich 312 Probanden an der Befragung. Davon wurden nach Selektion unvollständig ausgefüllter Fragebögen 250 Erhebungen in die Analyse einbezogen.

\subsection{Operationalisierung und Validierung der Konstrukte}

Die drei Konstrukte der Marken-Konsumenten-Beziehung sowie die Markenbindung werden im Modell reflektiv operationalisiert. Der Grund hierfür liegt darin, dass die Kausali- 
tätsrichtung von den Konstrukten zu den jeweiligen Items führt und die Konstruktitems voneinander abhängig und austauschbar sind. Zudem unterscheidet sich das nomologische Netz der Konstruktindikatoren nicht voneinander, da diesen dieselben Ursachen zugrunde liegen (Jarvis et al. 2003). Die Swissnessqualität und -quantität werden hingegen formativ operationalisiert, da deren Indikatoren jeweils unterschiedliche Facetten darstellen und somit nicht gegeneinander ausgetauscht werden können (Eberl 2006, 655; Albers/Hildebrand 2006, 11).

Die Massnahmen der Swissness bestehen aus den latenten Konstrukten Swissnessquantität und Swissnessqualität. Die Swissnessquantität (SQT) wird anhand von vier Items operationalisiert, die auf der Kontaktfrequenz und -intensität mit der Marke Ricola und der Schweiz beruhen. Auch die Operationalisierung der Swissnessqualität (SQL) basiert auf vier Items, die die Kongruenz zwischen der Marke Ricola und der Schweiz sowie die Qualität der kommunikativen Vermittlung der Swissness fokussieren. Da es sich um formative Konstrukte handelt, ist eine Überprüfung der internen Konsistenz, aufgrund der nicht notwendigen Abhängigkeit zwischen den Indikatoren nicht durchführbar. Die Messung der externen Validität ist nur durch eine sogenannte Phantomvariable (PV) möglich (Diamantopoulos/Winklhofer 2001, 272; Jarvis et al. 2003, 213). Dementsprechend werden die Konstrukte der Swissnessqualität und -quantität durch jeweils eine Phantomvariable mit einem Indikator reflektiv gemessen.

Die derivativ konzeptualisierte Marken-Konsumenten-Beziehung beinhaltet die drei eigenständigen Konstrukte Markenzufriedenheit, Markencommitment und Markenvertrauen. Diese latenten Konstrukte stellen die Beziehung zwischen der Marke Ricola und dem Konsumenten dar. Basierend auf Homburg et al. (1999, 392ff.) wird die Markenzufriedenheit (MZ) auf globaler Ebene über die Items Gesamtzufriedenheit mit der Marke, Grad der Erwartungserfüllung und Übereinstimmung mit einer Idealmarke abgefragt. Um das Konstrukt Markencommitment (MC) zu messen, werden in Anlehnung an Morgan und Hunt (1994, 29) die drei Indikatoren Verbundenheit zur Marke, Übereinstimmung mit dem Wertesystem der Marke und der konsumentenseitige Nutzen der Marke verwendet. Das Markenvertrauen (MV) wird ebenfalls über drei Items abgefragt. Entsprechend Delgado-Ballester et al. (2003, 41) werden das Vertrauen als gegenwartsgerichtete Komponente, das Vertrauen in zukünftige Leistungen sowie die vergangenheitsgerichtete Komponente, dass der Konsument von der Marke noch nie enttäuscht wurde, herangezogen.

Die Markenbindung (MB) kann grundsätzlich vergangenheitsgerichtet anhand des tatsächlichen Verhaltens oder zukunftsgerichtet über die Verhaltensabsicht abgefragt werden (Homburg et al. 2005, 1399). In dieser Arbeit wurde auf die gängigere ex ante Betrachtung - die Verhaltensabsicht - zurückgegriffen. Zur Operationalisierung werden in Anlehnung an die einschlägige Fachliteratur die Indikatoren Wiederkaufsabsicht, Cross-BuyingAbsicht, Weiterempfehlungsabsicht und Preiserhöhungsakzeptanz aufgegriffen (Homburg et al. 2005). Sämtliche Items sind in Tabelle 2 aufgeführt.

Im Rahmen der Gütebeurteilung der reflektiv gemessenen Konstrukte wurde in einem ersten Schritt anhand der exploratorischen Faktoranalyse bei allen Konstrukten eine eindimensionale Struktur nachgewiesen. Die Ladungen der exploratorischen Faktoranalyse liegen alle über dem geforderten Mindestwert von 0,7 bzw. 0,8 (Hulland 1999, 198). Der Varianzerklärungsbeitrag liegt bei jedem Konstrukt durch die ihm zugeordneten Indikatoren über den geforderten 50 Prozent, so dass sämtliche Items beibehalten werden (Chin 1998). Auch der erforderliche T-Wert von 1,64 wird für alle Items signifikant bestätigt. 


\begin{tabular}{|c|c|c|}
\hline Konstrukt & Code & Items \\
\hline \multirow{4}{*}{$\begin{array}{l}\text { Swissness- } \\
\text { quantität }\end{array}$} & Sqt1 & $\begin{array}{l}\text { Wenn ich Werbung der Marke Ricola sehe (in Zeitschriften, Fernsehen, } \\
\text { usw.), denke ich häufig an die Schweiz. }\end{array}$ \\
\hline & Sqt2 & $\begin{array}{l}\text { Ich nehme die Marke Ricola (im Handel, auf der Strasse, in Warenhäu- } \\
\text { sern/Kiosks, usw.) häufig als Schweizer Produkt wahr. }\end{array}$ \\
\hline & Sqt3 & $\begin{array}{l}\text { Wenn ich ein Bonbon der Marke Ricola konsumiere, nehme ich es oft als } \\
\text { Schweizer Produkt wahr. }\end{array}$ \\
\hline & Sqt4 & $\begin{array}{l}\text { Den Werbeslogan „Wer hat's erfunden? - Die Schweizer“ habe ich schon } \\
\text { oft gehört. }\end{array}$ \\
\hline \multirow{4}{*}{ Swissnessqualität } & Sql1 & Die Marke Ricola und die Schweiz passen gut zueinander. \\
\hline & $\mathrm{Sq} 12$ & $\begin{array}{l}\text { Die Bergwelt als Motiv auf der Verpackung von Ricola weist eindeutig } \\
\text { auf die Schweiz hin. }\end{array}$ \\
\hline & Sq13 & Die Produkte der Marke Ricola entsprechen der guten Schweizer Qualität. \\
\hline & Sql4 & Die Marke Ricola bekennt sich zum Standort Schweiz. \\
\hline \multirow{2}{*}{$\begin{array}{l}\text { Phantom- } \\
\text { variablen }\end{array}$} & Ph_Sqt & $\begin{array}{l}\text { Die Werbemassnahmen der Marke Ricola weisen eindeutig auf die } \\
\text { Schweiz hin. }\end{array}$ \\
\hline & Ph_Sql & Ich nehme die Marke Ricola als Schweizer Produkt wahr. \\
\hline \multirow{3}{*}{$\begin{array}{l}\text { Marken- } \\
\text { zufriedenheit }\end{array}$} & Mz1 & Mit der Marke Ricola bin ich insgesamt zufrieden. \\
\hline & $\mathrm{Mz} 2$ & Die Marke Ricola erfüllt meine Erwartungen an das Produkt. \\
\hline & Mz3 & Die Marke Ricola kommt einem idealen Produkt sehr nahe. \\
\hline \multirow{3}{*}{$\begin{array}{l}\text { Marken- } \\
\text { commitment }\end{array}$} & Mc1 & Ich fühle mich der Marke Ricola verbunden. \\
\hline & Mc2 & Ich kann mich gut mit der Marke Ricola identifizieren. \\
\hline & Mc3 & Die Marke Ricola bringt mir einen positiven Nutzen. \\
\hline \multirow{3}{*}{ Markenvertrauen } & Mv 1 & Ich vertraue der Marke Ricola. \\
\hline & Mv 2 & Die Marke Ricola hat mich noch nie enttäuscht. \\
\hline & Mv 3 & $\begin{array}{l}\text { Ich mache mir keine Sorgen bezüglich der zukünftigen Handlungen der } \\
\text { Marke Ricola. }\end{array}$ \\
\hline \multirow{4}{*}{ Markenbindung } & Mb1 & Ich entscheide mich auch in Zukunft für die Marke Ricola. \\
\hline & $\mathrm{Mb} 2$ & Ich werde auch andere Produkte der Marke Ricola ausprobieren/kaufen. \\
\hline & Mb3 & Ich werde die Marke Ricola weiterempfehlen. \\
\hline & Mb4 & $\begin{array}{l}\text { Ich beabsichtige ein Produkt der Marke Ricola zu kaufen, auch wenn eine } \\
\text { Preissteigerung stattfinden würde. }\end{array}$ \\
\hline
\end{tabular}

\section{Tabelle 2: Darstellung der verwendeten Items}

In einem zweiten Schritt ist die Konstruktreliabilität durch den Composite-ReliabilityWert zu betrachten. Es zeigt sich, dass alle Konstrukte den geforderten Mindestwert von 0,7 für Composite Reliability (CR) genügen. Das Cronbach'sche Alpha von mindestens 0,7 verweist auf ein hohes Mass an Reliabilität und Konvergenzvalidität. Ferner wurde anhand des Fornell-Larcker-Kriteriums die Diskriminanzvalidität überprüft. Die Auswertung erfolgte auf Basis des Analyseprogramms PLS Graph 3.0 (Chin et al. 2003). Es zeigt sich, dass die durchschnittlich erfasste Varianz (DEV) jedes Konstrukts über dem Grenzwert 
von 0,6 liegt. Da die DEV für alle Konstrukte grösser ist als die quadrierte Korrelation zwischen den Konstrukten, wird die Diskriminanzvalidität bestätigt (Fornell/Cha 1994, 72). Die Erfüllung des Fornell-Larcker-Kriteriums verweist somit darauf, dass die Markenzufriedenheit, das Markencommitment, das Markenvertrauen und die Markenbindung überschneidungsfreie Konstrukte darstellen. Die Ergebnisse zur Gütebeurteilung sind in den Tabellen 3 und 4 dargestellt.

\begin{tabular}{|c|c|c|c|}
\hline $\begin{array}{l}\text { Konstrukt } \\
\text { (reflektiv) }\end{array}$ & Indikator & $\begin{array}{l}\text { Faktorladung } \\
(\lambda>0,71 \text { bzw. } 0,8)\end{array}$ & t-Wert der Ladung \\
\hline \multirow{3}{*}{ Markenzufriedenheit } & Mz1 & 0,959 & $103,203 * *$ \\
\hline & Mz2 & 0,968 & $146,895 * *$ \\
\hline & Mz3 & 0,927 & $54,457 * * *$ \\
\hline \multirow{3}{*}{ Markencommitment } & Mc1 & 0,959 & $127,523 * * *$ \\
\hline & Mc2 & 0,954 & $149,376 * * *$ \\
\hline & Mc3 & 0,882 & $48,032 * * *$ \\
\hline \multirow{3}{*}{ Markenvertrauen } & Mv 1 & 0,916 & $81,621 * * *$ \\
\hline & Mv 2 & 0,916 & $68,223 * * *$ \\
\hline & Mv 3 & 0,861 & $30,875 * *$ \\
\hline \multirow{4}{*}{ Markenbindung } & $\mathrm{Mb} 1$ & 0,922 & $69,487 * * *$ \\
\hline & $\mathrm{Mb} 2$ & 0,867 & $30,331 * * *$ \\
\hline & Mb3 & 0,929 & $115,281 * * *$ \\
\hline & $\mathrm{Mb4}$ & 0,835 & $32,627 * * *$ \\
\hline $\begin{array}{l}\text { Konstrukt } \\
\text { (formativ) }\end{array}$ & Indikator & $\begin{array}{l}\text { Regressions- } \\
\text { koeffizient }(\beta>0,1)\end{array}$ & t-Wert der Regression \\
\hline \multirow{4}{*}{ Swissnessquantität } & Sqt1 & 0,216 & $2,312 *$ \\
\hline & Sqt2 & 0,359 & $3,423 * *$ \\
\hline & Sqt3 & 0,473 & $5,125 * * *$ \\
\hline & Sqt4 & 0,125 & $1,546^{\text {n.s. }}$ \\
\hline \multirow{4}{*}{ Swissnessqualität } & Sql1 & 0,193 & $1,987^{*}$ \\
\hline & $\mathrm{Sql2}$ & $-0,135$ & $1,818^{\text {n.s. }}$ \\
\hline & Sql3 & 0,838 & $10,249 * * *$ \\
\hline & Sql4 & 0,150 & $1,793^{\text {n.s. }}$ \\
\hline
\end{tabular}

Tabelle 3: Ergebnisse der Indikatorreliabilität für die Modellkonstrukte

Für formative Messmodelle sind aufgrund der umgekehrten Kausalitätsrichtung andere Gütekriterien heranzuziehen (Diamantopoulos/Winklhofer 2001, 271). Die Validität der Indikatoren wurde anhand multipler Regressionskoeffizienten überprüft. Als Mindestwert 
wird ein Indikatorgewicht von 0,1 angesehen (Jarvis et al. 2003, 202). Die Signifikanz der Regressionskoeffizienten wird anhand der t-Werte ermittelt. Die Validität wurde für alle Indikatoren der Swissnessquantität und -qualität bestätigt, ausser für den Indikator Sq12. Auch wenn einige Indikatoren nicht oder nur knapp nicht signifikant sind, werden diese beibehalten, da formative Konstrukte über die Gesamtheit ihrer Indikatoren definiert werden (Bollen/Lennox 1991, 308; Jarvis et al. 2003, 202). Letztlich wurde anhand der externen Validität nachgewiesen, dass die Indikatoren die formativen Konstrukte angemessen abbilden (Diamantopoulos/Winklhofer 2001, 273; Jarvis et al. 2003, 202). Hierfür wurde für die Swissnessquantität und -qualität jeweils eine reflektiv operationalisierte Phantomvariable genutzt. Der Mindestwert für den Pfadkoeffizienten von 0,5 wurde jeweils bestätigt (Diamantopoulos/Winklhofer 2001, 272f).

\begin{tabular}{|l|l|l|l|}
\hline $\begin{array}{l}\text { Konstrukt } \\
\text { (reflektiv) }\end{array}$ & $\begin{array}{l}\text { CR } \\
(>0,7)\end{array}$ & $\begin{array}{l}\text { Cronbach `sches Alpha } \\
(>0,7)\end{array}$ & $\begin{array}{l}\text { DEV } \\
(>0,6)\end{array}$ \\
\hline Markenzufriedenheit & 0,966 & 0,947 & 0,904 \\
\hline Markencommitment & 0,952 & 0,923 & 0,868 \\
\hline Markenvertrauen & 0,962 & 0,879 & 0,806 \\
\hline Markenbindung & 0,936 & 0,910 & 0,784 \\
\hline $\begin{array}{l}\text { Konstrukt } \\
\text { (formativ) }\end{array}$ & $\begin{array}{l}\mathrm{R}^{2} \\
(>0,33)\end{array}$ & $\begin{array}{l}\text { Pfadkoeffizient } \\
(>0,5)\end{array}$ & $\begin{array}{l}\text { t-Wert Pfad- } \\
\text { koeffizient }\end{array}$ \\
\hline $\begin{array}{l}\text { Phantomvariable der } \\
\text { Swissnessquantität }\end{array}$ & 0,525 & 0,511 & $5,451 * * *$ \\
\hline $\begin{array}{l}\text { Phantomvariable der } \\
\text { Swissnessqualität }\end{array}$ & 0,478 & 0,606 & $10,533 * * *$ \\
\hline
\end{tabular}

Tabelle 4: Gütebeurteilung der Messmodelle

\subsection{Schätzung des Kausalmodells und Modellbeurteilung}

Die Strukturgleichungsanalyse erfolgt ebenfalls auf Basis des Analyseprogramms PLS Graph 3.0. Zur Schätzung der Modellgüte, und damit zur Sicherstellung der Zuverlässigkeit des Gesamtmodells, wurde eine Orientierung an den von Chin vorgeschlagenen Prüfkriterien vorgenommen (Chin 1998; Chin et al. 2003). Die Gütebeurteilung des Strukturmodells erfolgt über das $\mathrm{R}^{2}$, das den durch die latenten exogenen Variablen erklärten Varianzanteil angibt. Chin $(1998,323)$ bezeichnet Werte über 0,33 als annehmbar. Im vorliegenden Modell ist die Varianzerklärung $\mathrm{R}^{2}$ für die Phantomvariablen der Swissnessquantität $(0,525)$ und Swissnessqualität $(0,478)$ sowie für die Konstrukte Markenzufriedenheit $(0,545)$, Markencommitment (0,479), Markenvertrauen $(0,551)$ und Markenbindung $(0,617)$ durchaus aussagekräftig. Somit ist zu konstatieren, dass eine genaue Messung der Swissnessquantität und -qualität, der zentralen Grössen der Marken-Konsumenten-Beziehung - Markenzufriedenheit, Markencommitment, Markenvertrauen - sowie der Markenbindung realisiert wurde. In Abbildung 2 und Tabelle 5 sind die Ergebnisse des Strukturgleichungsmodells dargestellt. 


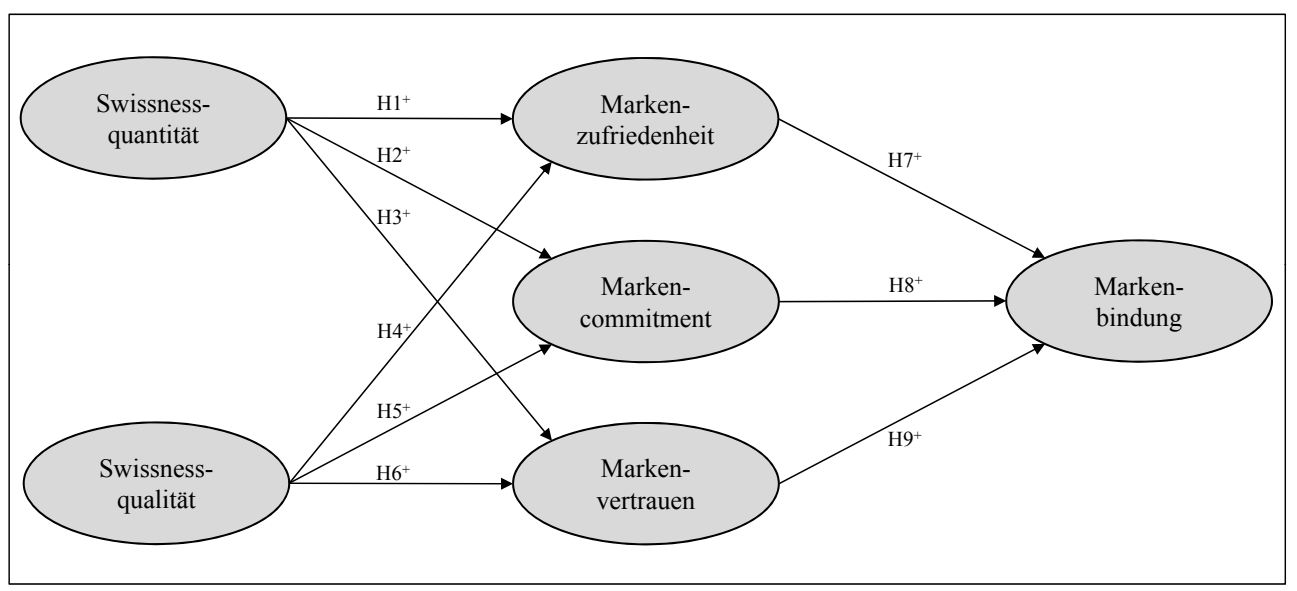

Abbildung 2: Ergebnisse des Strukturgleichungsmodells

Die Pfadkoeffizienten dienen zur Überprüfung der postulierten Hypothesen. Für die Beurteilung der Pfadkoeffizienten wird ein Mindestwert von 0,1 (Chin 1998, 324) gefordert. Sämtliche Pfadkoeffizienten weisen signifikante Werte von über 0,1 auf und sind daher als relevante Wirkungsbeziehungen zwischen den Konstrukten anzusehen.

\begin{tabular}{|l|l|l|}
\hline Konstrukt & $\mathrm{R}^{2}(>0,33)$ \\
\hline Markenzufriedenheit (MZ) & 0,545 & \multicolumn{2}{|l|}{} \\
\hline Markencommitment (MC) & 0,479 \\
\hline Markenvertrauen (MV) & 0,511 & \multicolumn{2}{|l|}{} \\
\hline Markenbindung (MB) & 0,617 & \multicolumn{2}{|l|}{} \\
\hline Swissnessquantität (SQT) & 0,525 & $\mathrm{t}$-Wert des Pfadkoeffizienten \\
\hline Swissnessqualität (SQL) & 0,478 & $2,488^{*}$ \\
\hline Pfadbeurteilung & Pfadkoeffizient $(>0,1)$ & $5,949^{* * *}$ \\
\hline SQT $\rightarrow$ MZ & 0,169 & $2,521^{* *}$ \\
\hline SQT $\rightarrow$ MC & 0,455 & $9,217^{* * *}$ \\
\hline SQT $\rightarrow$ MV & 0,180 & $3,921^{* * *}$ \\
\hline SQL $\rightarrow$ MZ & 0,608 & $8,556^{* * *}$ \\
\hline SQL $\rightarrow$ MC & 0,290 & $3,553^{* * *}$ \\
\hline SQL $\rightarrow$ MV & 0,603 & $8,991^{* * *}$ \\
\hline MZ $\rightarrow$ MB & 0,232 & $2,941^{* *}$ \\
\hline MC $\rightarrow$ MB & 0,447 & \\
\hline MV $\rightarrow$ MB & 0,202 &
\end{tabular}

Tabelle 5: Gütebeurteilung des Kausalmodells 


\subsection{Interpretation der Ergebnisse}

Im Rahmen der kausalanalytischen Prüfung des Modells an dem konkreten Beispiel wurden, wie in Tabelle 6 dargestellt, sämtliche Hypothesen bestätigt. Aus der vorliegenden Arbeit lassen sich folgende Ergebnisse ableiten. Die Bestätigung der Hypothesen 1, 2 und 3 weisen einen positiven Einfluss der Swissnessquantität auf die Beziehung des Konsumenten mit der Marke Ricola nach. Dabei ist vor allem der sehr starke Effekt der Swissnessquantität auf das Markencommitment hervorzuheben. Demnach wirkt ein häufiger Werbekontakt mit der Marke in Verbindung mit der Swissness besonders stark auf das Markencommitment als emotionale Komponente von Marken-Konsumenten-Beziehungen. Die Häufigkeit, mit der ein Konsument die Marke Ricola in Verbindung mit der Swissness wahrnimmt, hat des Weiteren einen signifikanten Einfluss auf seine Zufriedenheit mit der Marke Ricola und sein Vertrauen in diese Marke. Durch die Bestätigung der Hypothesen 4, 5 und 6 wird der Einfluss der Swissnessqualität auf die Marken-Konsumenten-Beziehung nachgewiesen. Insbesondere die beiden Pfadkoeffizienten von der Swissnessqualität auf die Markenzufriedenheit und das Markenvertrauen sind auffallend hoch. Erst bei der Betrachtung der Swissnessqualität auf Indikatorebene lassen sich plausible Rückschlüsse ziehen. Der Fokus wird dabei auf die beiden signifikanten Indikatoren Sq11 und Sql3 gelegt. Während sich Sql1 auf den „Fit“ bzw. die Kongruenz zwischen der Marke Ricola und der Schweiz als COO bezieht, berücksichtigt Sql3 inwieweit die wahrgenommene Produktqualität von Ricola der erwarteten Qualität Schweizer Produkte entspricht. Da die Bestätigung der erwarteten Produktqualität einen grossen Einfluss auf die Zufriedenheit eines Konsumenten hat, wird der starke Effekt der Swissnessqualität auf die Zufriedenheit mit der Marke Ricola nachvollziehbar. Auch für das Markenvertrauen ist die Produktqualität von Bedeutung. Zusätzlich wird das Markenvertrauen offensichtlich durch die Übertragung positiver Imagewerte des COO auf die Marke gestärkt.

\begin{tabular}{|l|l|c|}
\hline & Hypothese & \\
\hline H1 & Die Swissnessquantität hat einen positiven Einfluss auf die Markenzufriedenheit. & $\checkmark$ \\
\hline H2 & Die Swissnessquantität hat einen positiven Einfluss auf das Markencommitment. & $\checkmark$ \\
\hline H3 & Die Swissnessquantität hat einen positiven Einfluss auf das Markenvertrauen. & $\checkmark$ \\
\hline H4 & Je höher die Swissnessqualität ist, desto höher ist die Markenzufriedenheit. & $\checkmark$ \\
\hline H5 & Je höher die Swissnessqualität ist, desto grösser ist das Markencommitment. & $\checkmark$ \\
\hline H6 & Je höher die Swissnessqualität ist, desto grösser ist das Markenvertrauen. & $\checkmark$ \\
\hline H7 & $\begin{array}{l}\text { Je höher die Markenzufriedenheit eines Konsumenten ist, desto eher entwickelt } \\
\text { sich eine intensive Markenbindung. }\end{array}$ & $\checkmark$ \\
\hline H8 & $\begin{array}{l}\text { Je höher das Markencommitment eines Konsumenten ist, desto eher entwickelt } \\
\text { sich eine intensive Markenbindung. }\end{array}$ & $\checkmark$ \\
\hline H9 & $\begin{array}{l}\text { Je höher das Markenvertrauen eines Konsumenten ist, desto eher entwickelt sich } \\
\text { eine intensive Markenbindung. }\end{array}$ & $\checkmark$ \\
\hline
\end{tabular}

Tabelle 6: Ergebnisse der Hypothesenprüfung 
Die Hypothesen 7, 8 und 9 werden ebenfalls bestätigt. Demnach wirken die drei Konstrukte Markenzufriedenheit, Markencommitment und Markenvertrauen signifikant auf die Bindung des Konsumenten an die Marke Ricola. Von diesen Konstrukten ist der besonders starke Einfluss des Markencommitments auf die Markenbindung hervorzuheben. Das Markencommitment erfasst, wie stark sich Konsumenten mit einer Marke identifizieren und eine innere Verpflichtung gegenüber der Marke entwickeln. Erfolgreiche und langfristige Marken-Konsumenten-Beziehungen basieren demnach auf einer von Konsumenten freiwillig eingegangenen emotionalen Bindung an die Marke. Der konsumentenseitige Wunsch zur Bindung an eine Marke entsteht neben der Identifikation mit der Marke vorwiegend durch positive Erfahrungen mit sowie die Entwicklung von Vertrauen in die Marke.

Zusammenfassend ist festzuhalten, dass Massnahmen in Verbindung mit der Swissness die Marken-Konsumenten-Beziehung mit der Marke Ricola signifikant stärken, wobei die Swissnessquantität primär für das Markencommitment relevant ist und die Swissnessqualität in erster Linie die Markenzufriedenheit und das Markenvertrauen beeinflusst. Darüber hinaus wirken die Swissnessquantität und -qualität, über die Konstrukte der Marken-Konsumenten-Beziehung, positiv auf die Markenbindung.

\section{Fazit, Implikationen und Ausblick}

Aufgrund der zunehmenden Internationalisierung und dem damit einhergehenden Konkurrenzdruck stehen Unternehmen zunehmend vor der Herausforderung, Konsumenten langfristig an sich zu binden. Durch die Übertragung des Landesimages auf die Marke werden bei Konsumenten psychologische Wirkungen ausgelöst, welche die Markenbindung günstig beeinflussen. Die Zielsetzung der vorliegenden Studie war zum einen die Entwicklung eines Strukturgleichungsmodells unter Berücksichtigung der Wirkungsbeziehungen der Swissness auf psychologische und verhaltensbezogene Grössen insbesondere die zentralen Konstrukte der Marken-Konsumenten-Beziehung - Markenzufriedenheit, Markencommitment sowie Markenvertrauen - und der Markenbindung. Zum anderen setzte sich die vorliegende Studie zum Ziel, ein Modell zu entwickeln, das Rückschlüsse auf die Qualität der kommunikativen Übertragung der Swissness zulässt. Die empirische Überprüfung des Modells erfolgte am Beispiel der Schweizer Marke Ricola. Das Fallbeispiel führt zu folgenden Erkenntnissen: Die Ergebnisse der Untersuchung unterstreichen die Bedeutung der Swissnessquantität und Swissnessqualität. So fördern diese beiden Konstrukte eine hohe Beziehung und Bindung von Konsumenten an die Marke Ricola. Dies verweist auf die zentrale Rolle der Swissness für den Erfolg der Marke Ricola und verdeutlicht das Potenzial einer Swissnesspositionierung der Marke im Ausland.

Aus den Erkenntnissen lassen sich für die Marke Ricola folgende Implikationen für ein erfolgreiches internationales Markenmanagement ableiten:

Die Swissnessqualität wird hauptsächlich durch die Kongruenz der Marke mit der Schweiz und durch die Qualität der kommunikativen Übermittlung von COO-Informationen determiniert. Es ist folglich darauf $\mathrm{zu}$ achten, dass die Positionierung einer Marke wie im vorliegenden konkreten Beispiel - weiterhin in Verbindung mit der Schweiz steht, so dass ein Fit zwischen der Marke und der Schweiz gewährleistet wird. Ebenfalls ist ein regelmässiges Monitoring hinsichtlich der konsumentenseitigen Wahrnehmung der Schweiz zu betreiben, um bei eventuellen Veränderungen über entsprechende Modifikationen in der Positionierung der Marke nachzudenken. Um Assoziationen mit der Schweiz zu 
fördern, gilt es, die kommunikationspolitischen Massnahmen so auszugestalten, dass der Konsument auf die schweizerische Herkunft der Marke aufmerksam wird. Beispielsweise ist eine Produktmarkierung anhand Schweiz-typischer Merkmale erforderlich. Beim Einsatz der Swissness-Elemente ist darauf zu achten, dass die Botschaft von Konsumenten eindeutig mit der Schweiz in Verbindung gebracht wird. Zudem ist auf die Einhaltung der „guten Schweizer Qualität“ der Produkte der Marke hinzuweisen. So lassen sich im Zusammenhang mit der Swissness ausschliesslich qualitativ hochwertige Produkte glaubwürdig und erfolgreich vermarkten.

Eine erfolgreiche Übertragung des Landesimages auf die Marke Ricola verlangt ebenfalls die Berücksichtigung der Swissnessquantität. Die Sicherstellung einer hohen Swissnessquantität erfordert den permanenten und intensiven Einbezug von Swissness-Elementen in den Marketingmix einer internationalen Marke, wie z. B. Ricola. Dies gelingt durch eine konsequente Integration der Swissness über alle kommunikationspolitischen Massnahmen. So ist damit eine erhöhte Häufigkeit und Intensität, mit der die Swissness der Marke Ricola vermittelt wird, verbunden (vgl. hierzu die Ausführungen zur Integrierten Kommunikation von Brubn 2009).

Für die Wissenschaft zeigt der vorliegende Artikel weiteren Forschungsbedarf auf. Die Analyse des aktuellen Forschungsstands offenbart Defizite in der Untersuchung verhaltensbezogener und ökonomischer Wirkungsbeziehungen des COO. Zudem unterliegt die durchgeführte Studie einigen Restriktionen. Auch wenn das Modell anhand einer aussagekräftigen Stichprobe untersucht wurde, sind die Probanden vorwiegend deutscher Abstammung. Dementsprechend ist eine internationale Ausweitung der Erhebung durch die Befragung von Probanden aus unterschiedlichen Herkunftsländern empfehlenswert. Weiter befasst sich die Studie lediglich mit der Marke Ricola und der Konsumgüterbranche. Um den Aussagengehalt der Swissness und deren Wirkung auf die Grössen der Marken-Konsumenten-Beziehung und die Markenbindung zu erhöhen, ist die Berücksichtigung weiterer Marken und Branchen notwendig. Dies schafft die Grundlage für die Ableitung allgemeingültiger Implikationen für das internationale Markenmanagement von Unternehmen. Letztlich ergeben sich weitere Adaptionsmöglichkeiten durch die Ausweitung des Modells auf unterschiedliche Herkunftsländer, um die Gültigkeit des Modells über die Schweiz hinaus zu verallgemeinern.

Am Beispiel der Marke Ricola wurde in der vorliegenden Untersuchung gezeigt, dass die Swissness als Erfolgsfaktor der Markenführung dienen kann. Die Untersuchungsergebnisse führten zu ersten Erkenntnissen hinsichtlich der Wirkungsbeziehungen der Swissnessquantität und -qualität auf die Grössen der Marken-Konsumenten-Beziehung und die Markenbindung. Die Ergebnisse bieten eine gute Grundlage sowie Anregungen für weitere wissenschaftliche Untersuchungen.

\section{Literaturhinweise}

Aaker, J.L. (1997): Dimensions of Brand Personality, in: Journal of Marketing Research, Jg. 34, Nr. 4, S. 347-356.

Aaker, J.L./Fournier, S./Brasel, S.A. (2004): When Good Brands Do Bad, in: Journal of Consumer Research, Jg. 31, Nr. 1, S. 1-16.

Ahmed, Z.U./Johnson, J.P./Yang, X./Fatt, C.K./Teng, H.S./Boon, L.C. (2004): Does Country of Origin Matter for Low-Involvement Products?, in: International Marketing Review, Jg. 21, Nr. 1, S. 102-120. 
Albers, S./Hildebrandt, L. (2006): Methodische Probleme bei der Erfolgsfaktorenforschung - Messfehler, formative versus reflektive Indikatoren und die Wahl des Strukturgleichungsmodells, in: Zeitschrift für betriebswirtschaftliche Forschung, Jg. 58, Nr. 1, S. 2-33.

Altman, I./Taylor, D.A. (1973): Social Penetration. The Development of Interpersonal Relationships, New York.

Anderson, E./Weitz, B. (1992): The Use of Pledges to Build and Sustain Commitment in Distribution Channels, in: Journal of Marketing Research, Jg. 29, Nr. 1, S. 18-34.

Balabanis, G./Diamantopoulos, A. (2011): Gains and Losses from the Misperception of Brand Origin: The Role of Brand Strength and Country-of-Origin Image, in: Journal of International Marketing, Jg. 19, Nr. 2, S. 95-116.

Batra, R./Ramaswamy, V./Alden, D.L./Steenkamp, J.-B.E.M./Ramachander, S. (2000): Effects of Brand Local and Nonlocal Origin on Consumer Attitudes in Developing Countries, in: Journal of Consumer Psychology, Jg. 9, Nr. 2, S. 83-95.

Bauer, H.H./Mäder, R./Huber, F. (2002): Markenpersönlichkeit als Determinante von Markenloyalität, in: Zeitschrift für betriebswirtschaftliche Forschung, Jg. 54, Nr. 12, S. 687-709.

Bengtsson, A. (2003): Towards a Critique of Brand Relationships, in: Advances in Consumer Research, Jg. 30, S. 154-158.

Bilkey, W.J./Nes, E. (1982): Country-of-Origin Effects on Product Evaluations, in: Journal of International Business Studies, Jg. 13, Nr. 1, S. 89-99.

Bollen, K.A./Lennox, R. (1991): Conventional Wisdom on Measurement: A Structural Equation Perspective, in: Psychological Bulletin, Jg. 110, Nr. 2, S. 305-314.

Bruhn, M. (2009): Integrierte Unternehmens- und Markenkommunikation. Strategische Planung und operative Umsetzung, 5. Auflage, Stuttgart.

Casalo, L.V./Flavian, C./Guinaliu, M. (2008): Promoting Consumer's Participation in Virtual Brand Communities: A New Paradigm in Branding Strategy, in: Journal of Marketing Communications, Jg. 14, Nr. 1, S. 19-36.

Chaudhuri, A./Holbrook, M.B. (2002): Product-class Effects on Brand Commitment and Brand Outcomes: The Role of Brand Trust and Brand Affect, in: Journal of Brand Management, Jg. 10, Nr. 1, S. 33-58.

Chen, C.-W./Shen, C.-C./Chiu, W.-U. (2007): Marketing communication strategies in support of product launch: An empirical study of Taiwanese high-tech firms, in: Industrial Marketing Management, Jg. 36, Nr. 8, S. 1046-1056.

Chin, W.W. (1998): The Partial-Least-Squares Approach to Structural Equation Modelling, in: Marcoulides, G.A. (Hrsg.): Modern Methods for Business Research, Mahaw, S. 295-336.

Chin, W.W./Marcolin, B.L./Newsted, P.R. (2003): A Partial Least Squares Modeling Approach for Measuring Interaction Effects: Results from a Monte Carlo Simulation Study and an Electronic Mail Emotion/Adoption Study, in: Information Systems Research, Jg. 14, Nr. 2, S. 189-217.

Churchill, G.A./Surprenant, C. (1982): An Investigation in the Determinants of Customer Satisfaction, in: Journal of Marketing Research, Jg. 19, Nr. 10, S. 491-504.

Cordell, V.V. (1992): Effects of Consumer Preferences for Foreign Sourced Products, in: Journal of International Business Studies, Jg. 23, Nr. 2, S. 251-269.

Delgado-Ballester, E./Munuera-Alemán, J.L. (2001): Brand Trust in the Context of Consumer Loyalty, in: European Journal of Marketing, Jg. 35, Nr. 11/12, S. 1238-1258.

Delgado-Ballester, E./Munuera-Alemán, J.L./Yagüe-Guillén, M.J. (2003): Development and Validation of a Brand Trust Scale, in: International Journal of Market Research, Jg. 45, Nr. 1, S. 35-53. 
De Wulf, K./Odekerken-Schroder, G./Iacobucci, D. (2001): Investments in Consumer Relationships: A Cross-Country and Cross-Industry Exploration, in: Journal of Marketing, Jg. 65, Nr. 4, S. 33-50.

Diamantopoulos, A./Winklhofer, H.M. (2001): Index Construction with Formative Indicators: an Alternative to Scale Development, in: Journal of Marketing Research, Jg. 38, Nr. 2, S. 269-277.

Dibb, S./Meadows, M. (2004): Relationship Marketing and CRM: A Financial Services Case Study, in: Journal of Strategic Marketing, Jg. 12, Nr. 2, S. 111-125.

Eberl, M. (2006): Formative und reflektive Konstrukte und die Wahl des Strukturgleichungsverfahrens. Eine statistische Entscheidungshilfe, in: Die Betriebswirtschaft, Jg. 66, Nr. 6, S. 651-668.

Elliott, G.R./Cameron, R.C. (1994): Consumer Perception of Product Quality and the Country-ofOrigin Effect, in: Journal of International Marketing, Jg. 2, Nr. 2, S. 49-62.

Erickson, G.M./Johansson, J.K./Chao, P. (1984): Image Variables in Multi-Attribute Product Evaluations: Country of Origin Effects, in: Journal of Consumer Research, Jg. 11, Nr. 2, S. 694-699.

Esch, F.-R./Langner, T./Schmitt, B.H./Geus, P. (2006): Are Brands Forever? How Brand Knowledge and Relationships Affect Current and Future Purchases, in: Journal of Product \& Brand Management, Jg. 15, Nr. 2, S. 98-105.

Fang, X./Singh, S./Abluwalia, R. (2007): An Examination of Different Explanations for the Mere Exposure Effect, in: Journal of Consumer Research, Jg. 34, Nr. 1, S. 97-103.

Farrelly, F.J./Quester, P. (2005): Examining Important Relationship Quality Constructs of the Focal Sponsorship Exchange, in: Industrial Marketing Management, Jg. 34, Nr. 3, S. 211-219.

Feige, S./Brockdorff, B./Sausen, K./Fischer, P./Jaermann, U./Reinecke, S. (2008): Swissness Worldwide - Internationale Studie zur Wahrnehmung der Marke Schweiz, St. Gallen.

Feige, S./Fischer, P./Reinecke, S. (2010): Swissness Worldwide - Internationale Studie zur Wahrnehmung der Marke Schweiz, St. Gallen.

Fetscherin, M./Toncar, M. (2010): The Effects of the Country of Brand and the Country of Manufacturing of Automobiles: An Experimental Study of Consumers' Brand Personality Perceptions, in: International Marketing Review, Jg. 27, Nr. 2, S. 164-178.

Fleck, N.D./Quester, P. (2007): Birds of a Feather Flock Together... Definition, Role and Measure of Congruence: An Application to Sponsorship, in: Psychology \& Marketing, Jg. 24, Nr. 11, S. $975-1000$.

Fornell, C./Cha, J. (1994): Partial Least Squares, in: Bagozzi, R.P. (Hrsg.): Advanced Methods of Marketing Research, Oxford, S. 52-78.

Fournier, S. (1998): Consumer and Their Brands: Developing Relationship Theory in Consumer Research, in: Journal of Consumer Research, Jg. 24, Nr. 4, S. 343-373.

Fournier, S./Yao, J.L. (1997): Reviving Brand Loyalty: A Reconceptualization within the Framework of Consumer-Brand Relationships, in: International Journal of Research in Marketing, Jg. 14, Nr. 5, S. 451-472.

Garbarino, E./Johnson, M.S. (1999): The Different Roles of Satisfaction, Trust, and Commitment in Customer Relationships, in: Journal of Marketing, Jg. 63, Nr. 2, S. 70-87.

Gierl, H./Bartikowski, B. (2003): Ermittlung von Satisfiers, Dissatisfiers und Criticals in der Zufriedenheitsforschung, in: der markt, Jg. 42, Nr. 1, S. 14-34.

Grönroos, C. (2000): Creating a Relationship Dialogue: Communication, Interaction and Value, in: Marketing Review, Jg. 1, Nr. 1, S. 5-14.

Gounans, S.P. (2005): Trust and Commitment Influences on Customer Retention: Insights from Business-to-Business Services, in: Journal of Business Research, Jg. 58, Nr. 2, S. 126-140. 
Gürhan-Canli, Z./Maheswaran, D. (2000a): Cultural Variations in COO Effects, in: Journal of Marketing Research, Jg. 37, Nr. 3, S. 309-317.

Gürhan-Canli, Z./Maheswaran, D. (2000b): Determinants of Country-of-Origin Evaluations, in: Journal of Consumer Research, Jg. 27, Nr. 1, S. 96-108.

Han, C.M. (1989): Country Image: Halo or Summary Construct?, in: Journal of Marketing Research, Jg. 26, Nr. 2, S. 222-229.

Hastak, M./Hong, S.-T. (1991): Country-of-Origin Effects on Product Quality Judgments: An Information Integration Perspective, in: Psychology \& Marketing, Jg. 8, Nr. 2, S. 129-143.

Heimbach, A.E./Johansson, J.K./MacLachlan, D.L. (1989): Product Familiarity, Information Processing, and Country-of-Origin Cues, in: Advances in Consumer Research, Jg. 16, Nr. 1, S. 460-467.

Homburg, Ch./Fassnacht, M./Werner, H. (1999): Operationalisierung von Kundenzufriedenheit und Kundenbindung, in: Bruhn, M./Homburg, Ch. (Hrsg.): Handbuch Kundenbindungsmanagement. Grundlagen - Konzepte - Erfahrungen, 2. Auflage, Wiesbaden.

Homburg, Ch./Giering, A. (1996): Konzeptualisierung und Operationalisierung komplexer Konstrukte, in: Marketing ZFP, Jg. 18, Nr. 1, S. 5-24.

Homburg, Ch./Koschate, N./Becker, A. (2005): Messung von Markenzufriedenheit und Markenloyalität, in: Esch, F.-R. (Hrsg.): Moderne Markenführung. Grundlagen - Innovative Ansätze Praktische Umsetzungen, 4. Auflage, Wiesbaden, S. 1393-1408.

Hong, S.-T./Kang, D.K. (2006): Country-of-Origin Influences on Product Evaluations: The Impact of Animosity and Perceptions of Industriousness Brutality on Judgments of Typical and Atypical Products, in: Journal of Consumer Psychology, Jg. 16, Nr. 3, S. 232-239.

Hong, S.-T./Wyer Jr., R.S. (1989): Effects of Country-of-Origin and Product-Attribute Information on Product Evaluation: An Information Processing Perspective, in: Journal of Consumer Research, Jg. 16, Nr. 2, S. 175-187.

Hong, S.-T./Wyer Jr., R.S. (1990): Determinants of Product Evaluation: Effects of the Time Interval between Knowledge of a Product's Country of Origin and Information about its Specific Attributes, in: Journal of Consumer Research, Jg. 17, Nr. 3, S. 277-288.

Hulland, J. (1999): Use of Partial Least Squares (PLS) in Strategic Management Research: A Review of Four Recent Studies, in: Strategic Management Journal, Jg. 20, Nr. 2, S. 195-204.

Iacobucci, D./Hibbard, J.D. (1999): Toward an Encompassing Theory of Business Marketing Relationships (BMRs) and Interpersonal Commercial Relationships (ICRs): An Empirical Generalization, in: Journal of Interactive Marketing, Jg. 13, Nr. 3, S. 13-33.

Janda, S./Rao, C.P (1997): The Effect of Country-of-Origin Related Stereotypes and Personal Beliefs on Product Evaluation, in: Psychology \& Marketing, Jg. 14, Nr. 7, S. 689-702.

Jarvis, Ch.B./MacKenzie, S.B./Podsakoff, Ph.M. (2003): A Critical Review of Construct Indicators and Measurement Model Misspecification in Marketing and Consumer Research, in: Journal of Consumer Research, Jg. 30, Nr. 2, S. 199-218.

Jiménez, N.H./San Martín, S. (2010): The Role of Country-of-Origin, Ethnocentrism and Animosity in Promoting Consumer Trust. The Moderating Role of Familiarity, in: International Business Review, Jg. 19, Nr. 1, S. 34-45.

Johansson, J.K./Douglas, S.P./Nonaka, I. (1985): Assessing the Impact of Country of Origin on Product Evaluations: A New Methodological Perspective, in: Journal of Marketing Research, Jg. 22, Nr. 4, S. 388-396.

Kühn, R. (1992): Das „Made-in-Image“ der Schweiz als strategischer Parameter, in: Die Unternehmung, Jg. 46, Nr. 4, S. 303-314. 
Kühn, R./Weiss, M. (1998): Das „Made-in“-Image der Schweiz im internationalen Vergleich, in: Die Unternehmung, Jg. 52, Nr. 1, S. 49-60.

Lee, J.K./Lee, W.-N. (2009): Country-of-Origin Effects on Consumer Product Evaluation and Purchase Intention: The Role of Objective versus Subjective Knowledge, in: Journal of International Consumer Marketing, Jg. 21, Nr. 2, S. 137-151.

Li, W.-K./Leung, K./Wyer Jr., R.S. (1993): The Roles of Country of Origin Information on Buyers' Product Evaluations: Signal or Attribute?, in: Advances in Consumer Research, Jg. 20, Nr. 1, S. 684-689.

Li, W.-K./Monroe, K.B./Chan, D.K.-S. (1994): The Effects of Country of Origin, Brand, and Price Information: A Cognitive-Affective Model of Buying Intentions, in: Advances in Consumer Research, Jg. 21, Nr. 1, S. 449-457.

Li, W.-K./Wyer Jr., R.S. (1994): The Role of Country of Origin in Product Evaluations: Informational and Standard-of-Comparison Effects, in: Journal of Consumer Psychology, Jg. 3, Nr. 2, S. 187-212.

Liefeld, J.P. (2004): Consumer Knowledge and Use of Country-of-Origin Information at the Point of Purchase, in: Journal of Consumer Behaviour, Jg. 4, Nr. 2, S. 85-96.

Liu, S.S./Johnson, K.F. (2005): The Automatic Country-of-Origin Effects on Brand Judgments, in: Journal of Advertising, Jg. 34, Nr. 1, S. 87-97.

Madden, T.J./Fehle, F./Fournier, S. (2006): Brands Matter: An Empirical Dem-onstration of the Creation of Shareholder Value Through Branding, in: Journal of the Academy of Marketing Science, Jg. 34, Nr. 2, S. 224-235.

Maheswaran, D. (1994): Country of Origin as a Stereotype: Effects of Consumer Expertise and Attribute Strength on Product Evaluations, in: Journal of Consumer Research, Jg. 21, Nr. 2, S. 354-365.

Maheswaran, D./Chen, C.Y. (2006): Nation Equity: Incidental Emotions in Country-of-Origin Effects, in: Journal of Consumer Research, Jg. 33, Nr. 3, S. 370-376.

Michaelis, M./Woisetschläger, D./Backhaus, Ch./Ablert, D. (2008): The Effects of Country of Origin and Corporate Reputation on Initial Trust: An Experimental Evaluation of the Perception of Polish Consumers, in: International Marketing Review, Jg. 25, Nr. 4, S. 404-422.

Morgan, R.M./Hunt, S.D. (1994): The Commitment-Trust Theory of Relationship Marketing, in: Journal of Marketing, Jg. 58, Nr. 3, S. 20-38.

Oliver, R.L./DeSarbo, W.S. (1988): Response Determinants in Satisfaction Judgments, in: Journal of Consumer Research, Jg. 14, Nr. 4, S. 495-507.

Parameswaran, R./Pisharodi, R.M. (1994): Facets of Country of Origin Image: An Empirical Assessment, in: Journal of Advertising, Jg. 23, Nr. 1, S. 43-56.

Peterson, R.A. (2001): On the Use of College Students in Social Science Research: Insights from a Second-Order Meta-analysis, in: Journal of Consumer Research, Jg. 28, Nr. 3, S. 450-462.

Pisharodi, R.M./Parameswaran, R. (1992): Confirmatory Factor Analysis of Country-of-Origin Scale: Initial Results, in: Advances in Consumer Research, Jg. 19, Nr. 1, S. 706-714.

Romaniuk, J./Sharp, B. (2003): Measuring brand perceptions: Testing quantity and quality, in: Journal of Targeting, Measurement \& Analysis for Marketing, Jg. 11, Nr. 3, S. 218-229.

Roth, M.S./Romeo, J.B. (1992): Matching Product Category and Country Image Perceptions: A Framework for Managing Country-of-Origin Effects, in: Journal of International Business Studies, Jg. 23, Nr. 3, S. 477-497. 
Samiee, S./Shimp, T.A./Sharma, S. (2005): Brand origin recognition accuracy: its antecedents and consumers' cognitive limitations, in: Journal of International Business Studies, Jg. 36, Nr. 4, S. 379-397.

Swaminathan, V./Page, K.L./Gürhan-Canli, Z. (2007): „My“ Brand or „Our“ Brand: The Effects of Brand Relationship Dimensions and Self-Construal on Brand Evaluations, in: Journal of Consumer Research, Jg. 34, Nr. 2, S. 248-259.

Taylor, S.E./Peplau, L.A./Sears, D.O. (2002): Social Psychology, New York.

Thakor, M.V./Lavack, A.M. (2003): Effect of perceived brand origin associations on consumer perceptions of quality, in: Journal of Product \& Brand Management, Jg. 12, Nr. 6, S. 394-407.

Thorelli, H.B./Lim, J./Ye, J. (1989): Relative Importance of Country of Origin, Warranty and Retail Store Image on Product Evaluations, in: International Marketing Review, Jg. 6, Nr. 1, S. 35-46.

Till, B.D./Busler, M. (2000): The Match-up Hypothesis: Physical Attractiveness, Expertise, and the Role of Fit on Brand Attitude, Purchase Intent and Brand Beliefs, in: Journal of Advertising, Jg. 29, Nr. 3, S. 1-13.

Tse, D.K./Gorn, G.J. (1993): An Experiment on the Salience of Country-of-Origin in the Era of Global Brands, in: Journal of International Marketing, Jg. 1, Nr. 1, S. 57-76.

Usunier, J.-C./Cestre, G. (2007): Product Ethnicity: Revisiting the Match Between Products and Countries, in: Journal of International Marketing, Jg. 15, Nr. 3, S. 32-72.

Veloutsou, C. (2007): Identifying the Dimensions of the Product-Brand and Consumer Relationship, in: Journal of Marketing Management, Jg. 23, Nr. 1/2, S. 7-26.

Verlegh, P.W.J./Steenkamp, J.E.M. (1999): A review and meta-analysis of country-of-origin research, in: Journal of Economic Psychology, Jg. 20, Nr. 5, S. 521-546.

Walter, A./Müller, T.A./Helert, G./Ritter, T. (2003): Functions of industrial supplier relationships and their impact on relationship quality, in: Industrial Marketing Management, Jg. 32, Nr. 2, S. 159-169.

Zajonc, R.B. (1968): Attitudinal Effects of Mere Exposure, in: Journal of Personality and Social Psychology Monograph Supplement, Jg. 9, Nr. 2, S. 1-27.

Manfred Bruhn, Prof. Dr., ist Inhaber des Lehrstuhls für Betriebswirtschaftslehre, insbesondere Marketing und Unternehmensführung, an der Wirtschaftswissenschaftlichen Fakultät der Universität Basel, und Honorarprofessor an der Technischen Universität München.

Jürgen Schwarz, Dipl.-Kfm., ist wissenschaftlicher Mitarbeiter am Lehrstuhl für Betriebswirtschaftslehre, insbesondere Marketing und Unternehmensführung, an der Wirtschaftswissenschaftlichen Fakultät der Universität Basel.

Verena Batt, Dipl.-Rom., ist wissenschaftliche Mitarbeiterin am Lehrstuhl für Betriebswirtschaftslehre, insbesondere Marketing und Unternehmensführung, an der Wirtschaftswissenschaftlichen Fakultät der Universität Basel.

Anschrift: Universität Basel, Wirtschaftswissenschaftliche Fakultät, Lehrstuhl für Marketing und Unternehmensführung, Peter Merian-Weg 6, CH-4002 Basel, Tel.: +41 (0)61/267-32-23, Fax: +41 (0)61/267-28-38, E-Mails: manfred.bruhn@unibas.ch, juergen.schwarz@unibas.ch, verena.batt@unibas.ch 